\title{
Aspect of QOL Assessment and Proposed New Scale for Evaluation
}

\author{
Nobuo Yamaguchi1,2*, Matsuo Arai2, Tsugiya Murayama ${ }^{2,3}$ \\ ${ }^{1}$ Department of Fundamental Research for CAM, Kanazawa Medical University, Ishikawa, Japan \\ ${ }^{2}$ Ishikawa Natural Medicinal Products Research Center, Ishikawa, Japan \\ ${ }^{3}$ Faculty of Pharmaceutical Sciences, Department of Microbiology and Immunology, Hokuriku University, \\ Ishikawa, Japan

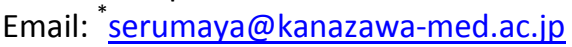

Received 14 May 2015; accepted 28 August 2015; published 31 August 2015

Copyright (C) 2015 by authors and Scientific Research Publishing Inc.

This work is licensed under the Creative Commons Attribution International License (CC BY). http://creativecommons.org/licenses/by/4.0/

(c) (i) Open Access

\section{Abstract}

According to the reports from Ministry of Health in each country, the average life span is elongated over the past decade. This trend is expected to be keeping constant further in the future and at the same time continued efforts should be made. Next, it is necessary for us to consent about improving the quality of life. In an effort of improving the quality of life, other than the western medicine, we have attempted to introduce many traditional medical practices, including the oriental medicine, from various parts of the world into the medical field as a complementary \& alternative medicine (CAM). Judging both positive and negative aspects by the evaluation standard of the Western medicine, we try to obtain numerical aspects in termed of QOL. Some methods of alternative medicine involved in this review are acupuncture, moxibustion, TCM and other traditional medicine. Above all, WHO suggests selecting the suzerain nation of acupuncture moxibustion, which has been developed in the oriental countries as a specialized cure, by identifying merits \& demerits and ascertaining which nation can be a the best model for special field. At the moment for evaluating CAM, for example, what kind of methods is/are suitable for evaluating each CAM. We have been trying to propose the peripheral leukocyte, that is one of the best marker for evaluating CAM. Our trial is a hot spring hydrotherapy, acupuncture and moxibution, light exercise and Ondle/floor heating etc. In these trials, we find the common results from leukocyte effects which exhibit a strong correlation for the regulation in number and function, as a new scale for evaluating QOL. The contents are the result from the data showing the much in number of volunteer tend to down regulate, on the other hand, lower number of volunteer is up-regulated at next day by each menu of CAM as a constitution dependent manner. However, even in western medical methodology, double blind and cross over system are the better way to evaluation even in CAM. The responsibility in human to each CAM therapy is different in individually, so to say according to constitution. In other words, there are so many vectors within the group, including at least three

${ }^{*}$ Corresponding author. 
groups, up-regulating, down regulating and stand stilled one. So we have no positive results by the method by sum-up and made mean. According to this system, individual vector is cancelled each other and real effect is not exhibited as a final result. So, we would like to present more smart way to access the efficacy for CAM menu. That is trial to plot each individual effect on the linear function and making slant/co-efficiency that plot each variable vector which is derived from the relative value compared to that of the day before. This procedure is easy to compare between each impact to the volunteer. We would like to propose this evaluation system as Super Constitution System: SCS. This kind of regulation showed within a 24 hours, for the leukocyte subsets, granulocyte and lymphocyte are changed even if they are under control of the circadian rhythm. So, for the purpose of evaluating leukocyte deviation, we set the point for evaluation at the same time-zone from the first set of evaluation to the next time-zone of evaluation. Under these conditions, we get a same result reproductively that the whole number of leukocyte, and its subset, granulocyte and lymphocyte also regulate within a 24 hours. This kind of phenomenon is the case that we confirm a lot of kind of CAM with repetition. This kind of regulation is confirmed in the content of the emotional hormone regulation. Within a hormonal change, adrenalin and dopamine are just under the regulation in the mode of SCS. However, the life span of leukocyte are at lease 3 - 4 days in rapid group of leukocytes, and no such a drastic apoptosis is induced by such a stimulation. For the purpose of scientific explanation, we also propose that the emotional hormone is concerned this change of leukocytes population. For the results of this hypothesis, there are reasonable changes in the peripheral blood about emotional hormone, adrenaline and dopamine. Other hormone concern thyroid grand is not changed significantly. In the final part of this chapter, we also propose that the suitable approach for anti-oxidative assessment and the effective pathway of complement for enabling some historical food supplement activate the whole body cell that expresses a complement receptor. This is not a case of infection but another stage of complement activation by fragmented polysaccharides for historical human use as foods. The protein materials are also fermented and served as digestive food around the world. Almost all the products have some distinctive smell but accompanying some effects through independent digestive rout for intact protein itself. With these aspects, we try to emphasize SCS and discuss by showing evident based manner with various menu of CAM.

\section{Keywords}

QOL, Leukocyte Subset, Granulocyte, Lymphocyte, Constitution Dependent Assay System, Double Blind-Cross Over System, Immunological Factors, Emotional Hormone, Adrenalin, Dopamine, Anti-Oxidative Assay by Macrophage, Immunological Factors

\section{Introduction}

There are two primary systems: innate and adaptive. Despite this defenses system in the overwhelming problems of possessing this dual system, the innate and adoptive do not seem to guard or even prevent the development of one internal threat to survival, However, every individual in the world exposes to the lisk of immunodeficiency in daily life with both internal and externals. The factors that influence the acquired immune activity are systemic metabolic disorder such as diabetes mellitus, malnutrition, extreme exhaustion, stress, aging and medical side effect in cancer [1]-[12]. So we have to select appropriate menu to regulate immune function through leukocyte storage. The menu has been summarized and listed as CAM: complementary and alternative medicine.

In this review, we plan to collect evidence and judge them with the content suggested in the case of eCAM setup. In other words, as a judging standard, setting immunologic factors as main items, we will judge superior and inferior of methods in each country.

Recommending not only quantitatively, but also qualitatively to evaluate "balance of the lymphocyte which is the associate of the white blood corpuscle and the polymorph" as a standard of the alternative medicine.

Every creature in the world including human exposes to the lisk of immunodeficiency in daily life [1]-[10]. The factors that influence the acquired immune activity are systemic metabolic disorder such as diabetes meritus, 
mal-nutrition, extreme exhaustion and stress, senile and side effect in cancer. So we have to select a daily appropriate menu to regulate immune function through leukocyte storage. The menu has been summarized and listed as CAM: complementary and alternative medicine. One of the major menus is TCM in western medicine world, some trying to integrate Western Medicine and Eastern Medicine (Figure 1).

\section{The Urgent Need for QOL Assessment}

1) A choice of health menu in conjunction with each constitution is in a quandary due to lack of information concerning these cross-interactions among general public and lack of information among the health professionals resulting with a potentially significant health scale.

2) A vertebrate animal acquired two ontogenically and phylogenically defense systems and ontogenetically, innate and adaptive. Despite these defense systems overwhelming problems of possessing these dual systems, the innate and adoptive does not seem to guard or even prevent the development of one internal threat to survival. However, every individual expose to the risks of immunodeficiency status in daily life with both internal and externals [1]-[12]. The factors that influence the acquired immune activity are systemic metabolic disorder such as diabetes mellitus, malnutrition, extreme exhaustion, extensive stress, aging and medical side effects [1]-[12]. So we have to select appropriate menu to regulate immune function through leukocyte storage.

In other words, no tentative scale for evaluate the intense of each trial. We have been reported the best tailored scale by different menu through leukocyte regulation in number and function [13]. We have been trying to regulate the immune responsiveness through much mature for fragile in daily stress and so on. The main menu from our trials were, acupuncture, hot-spring hydrotherapy, light exercise Ondel heating etc. In this article, we would like to show the regulatory mechanism of the hot spring hydrotherapy. The circumstance, the balneotherapy using the effectiveness of hot-springs hydrotherapy, except for cases of contraindication, has been medically useful approved to be effective in many stress-related disorders and the improvement of dysfunction of the biological rhythm disturbance as well as chronic disease. The mechanism of effects has been reported in many studies, but many things are still unclear. Balneotherapy needs to be treated in general a period of time, but the

\section{Aquired Immuno Deficiencies}

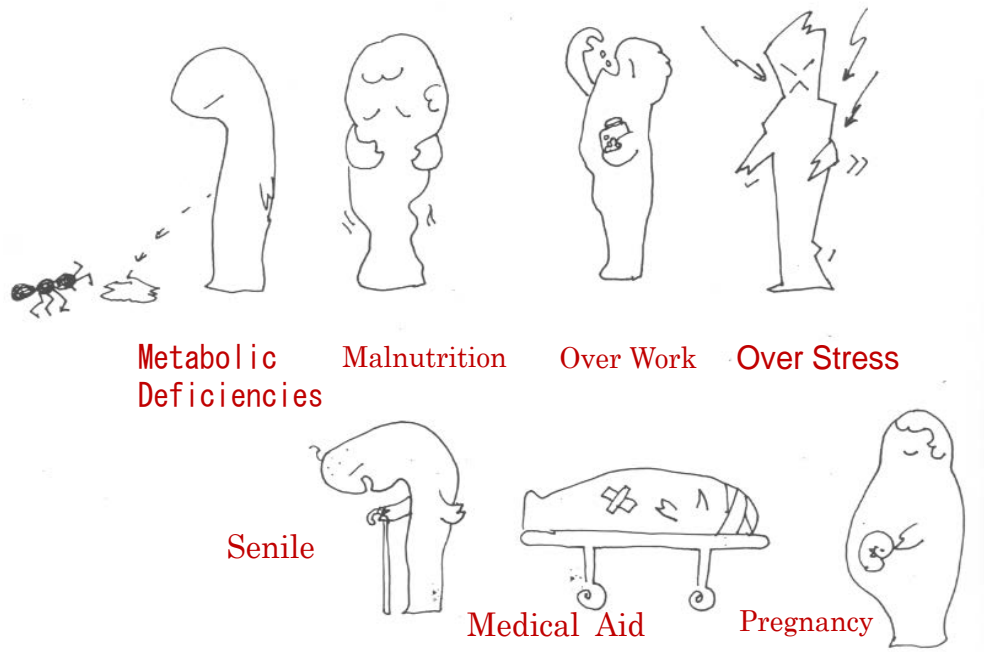

Figure 1. Acquired Immunodeficiency in Daily Life. The lists for causative factors induce acquired immune-deficiency.in daily life. We had prepared the native non-specific gourd line outside of the skin and/or mucous membrane. However, the gourd line is easily broken by accidental affair. However, nonspecific and specific attack system had been prepare as lympho-reticular line of defuse. This defense lines are accidentally broken by the factor listed in this figure. 
effectiveness has been suggested even if the short duration hot-springs hydrotherapy. We examined the effect of hot spring hydrotherapy for a short duration on immune system and reported about the quantitative and qualitative variation of immuno-competent cells [11]-[14]. The mechanism is suggested the association with an autonomic nervous system and the endocrine system. Hot spring hydrotherapy for a short duration was expected to stimulate sympathetic nerve or parasympathetic nerve and to change the levels of catecholamines (adrenalin, noradrenaline, and dopamine), which are neurotransmitters and hormones, as well as the number and function of immune cells (Figure 2).

\section{A Simple Sum-Up \& Make Mean Fade out the Important Changes, in a System Double-Blind \& Cross over}

There are many experimental system for evaluating QOL on the basis of western medicine. Almost all the experimental protocols are recommended to double blind and cross over system as a better evaluating system. However, simple sum up and make mean for comparison of efficacy before and after the administrating some menu. Our evidence from light exercise, walking, hot spring hydrotherapy etc., we had no result by the method, making summing up and make mean in all the menu of CAM (Figure 3). For at least three types of individuals that responded to up-regulation, down regulation and no changed one. So simple summing up is cancelled the each vector of individual. The $\mathrm{X}$-axis according to the value before each CAM menu. As shown in the Figures, the data could represent by linear slant. The value correlation/tangent was represented the each result from CAM menu/walking. We tried to compare the best impact for each individual, we set up two sort of impact. The one was walking $4 \mathrm{~km} /$ hour (4 mets), and the other was $8 \mathrm{~km} /$ hour ( 8 mets) to the same volunteer at the different day after the cooling of the former menu (one month interval).

In order to establish some effect from each designed experiment, one usually recommended to make experimental system as double blind and crossing over system. Hot Spring Hydrotherapy Regulate Peripheral Leukocyte Together with Emotional Hormone and Receptor Positive Lymphocytes According to Each Constitu-

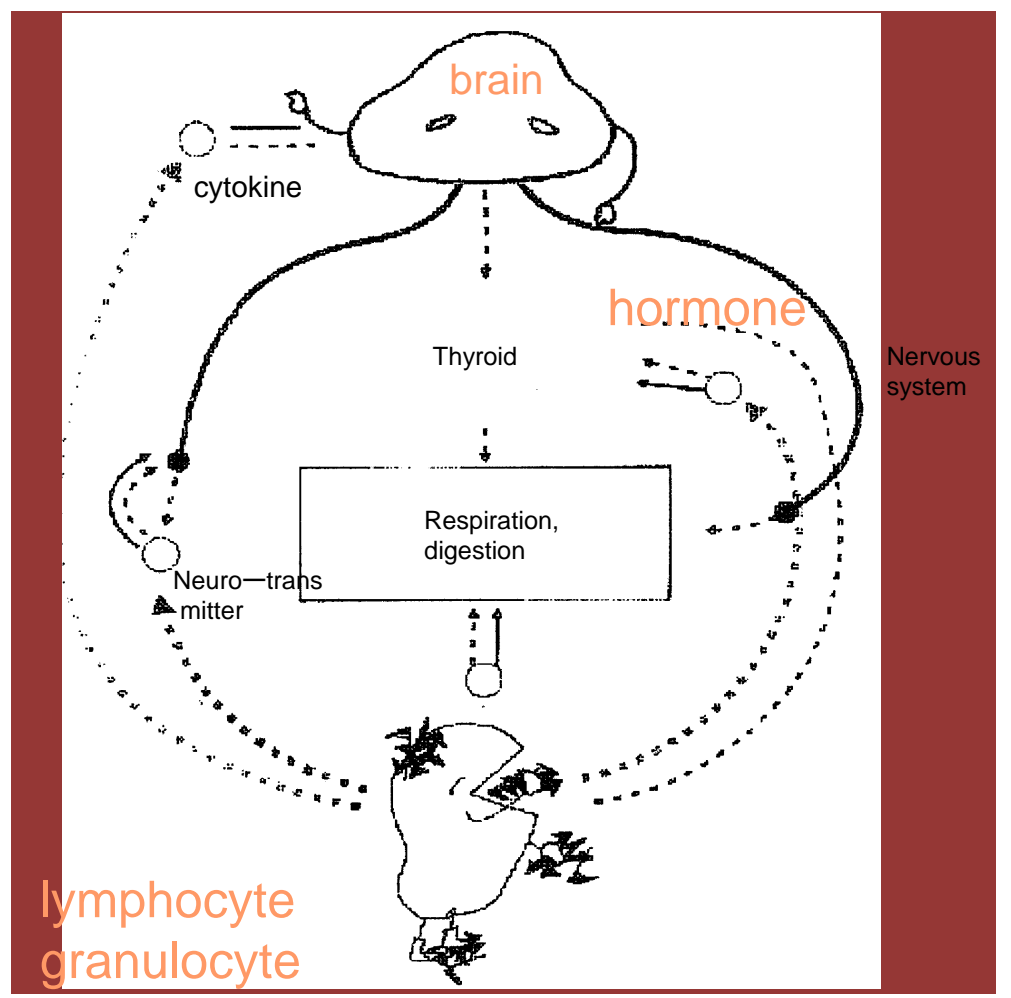

Figure 2. The illustration showed a close network between brain, hormone and peripheral leukocyte. In this review, peripheral leukocyte and hormone level were tie-up each other so to in a constitution dependent manner, especially in emotional hormone, adrenalin and dopamine. 


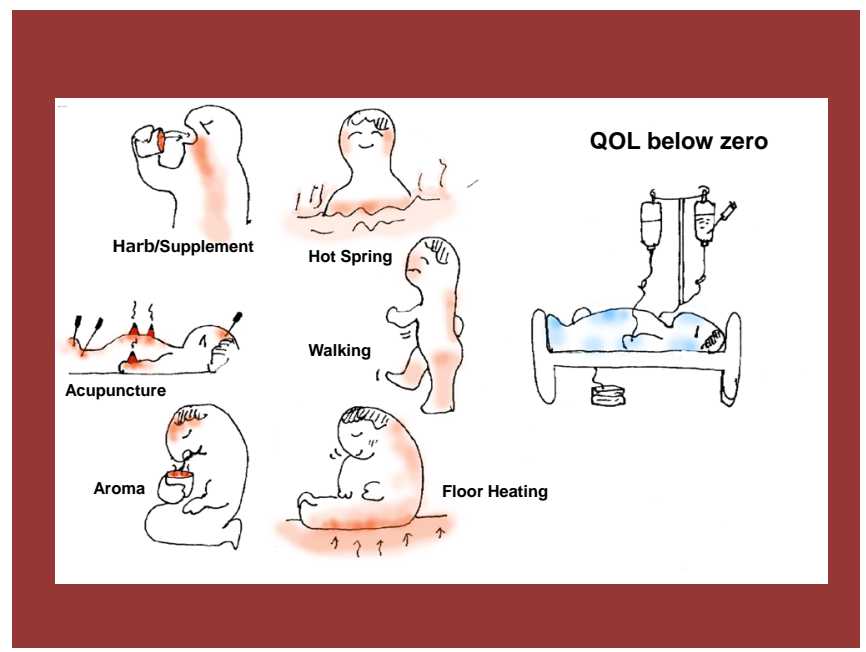

Figure 3. The CAM menu that introduced in this article with evidence based manner on the basis of each constitution/condition. We tried to express the effect of peripheral total leukocyte number by individual level of change and plot in the X-axis as in each age. The confirmed menu were, hot-spring hydrotherapy, supplement administration especially with fomented polysaccharide and depredated protein, light exercise, acupuncture, floor heating and aroma therapy Variations in leukocyte subpopulations in the peripheral blood before and after each CAM therapy.

tion/Condition is important to conscious of circadian rhythm. Abo reported that it was possible to sort the constitution, granulocyte-rich individual and lymphocyte-rich one with the peripheral leukocyte [15]-[26].

Each population of subset is depending on a circadian rhythm. Within a same individual, granulocyte increase in the daytime, on the other hand, lymphocyte increased in the nighttime in a cycle 24 hrs. So we have to compare the effect of each menu for the peripheral leukocyte on the same time before and after the menu. Figures 3-6.

\section{The Best Scale of QOL Assessment for Constitutionally Different in Human}

Our results showed that within 24 hours after hot spring hydrotherapy, the white blood cells in peripheral blood had changed significantly, not only in cell count but also cell function. We hope that our work will attract more attention to the mechanisms of which hot spring hydrotherapy regulates the human immune system. Abo reported that according to the lymphocyte subset content, lymphocyte rich type showed over $40 \%$ on the other hand granulocyte rich type show over $60 \%$ of granulocyte [27] [28]. Each type exhibited different character even in the same age, sexuality and each age. In the Figures, within the same age and the sex, even in mankind can sorts out as G-rich type (granulocyte 60\%), and L-rich type (lymphocyte 40\%). On the other hand, as a stand point of sex difference, the lady belongs to L-rich type.

Hot Spring Hydrotherapy Regulate Peripheral Leukocyte Together with Emotional Hormone and Receptor Positive Lymphocytes according to each constitution/condition but the gentleman belongs G-rich type. According to the age-related change, G-rich type of young man change to L-rich type according getting older.

We have been trying to regulate the immune responsiveness through much mature for fragile daily condition from circumstance stress and so on. The main menu were, acupuncture, hot-spring hydrotherapy, light exercise etc. In this article, we would like to show the regulatory mechanism of the hot spring hydrotherapy.

As shown in the Figure 7 and Figure 8, Leucocyte subsets, granulocyte and Lymphocyte are regulated by each CAM menu. Moreover, we tried to select emotional hormone in order to catch a possibility to show regulational factor dependent on each condition and constitution. We had selected 5 hormones for possible candidate, adrenalin, nor-adrenalin dopamine, ACTH, T4 and T5, adrenalin and dopamine were completely dependent on the condition and constitution.

In the Figures, the regulational bias was also could indicated as slant, correlation efficiency, possible to express one key word of number, easy to compare the efficacy of each menu. For example, we selected the light 


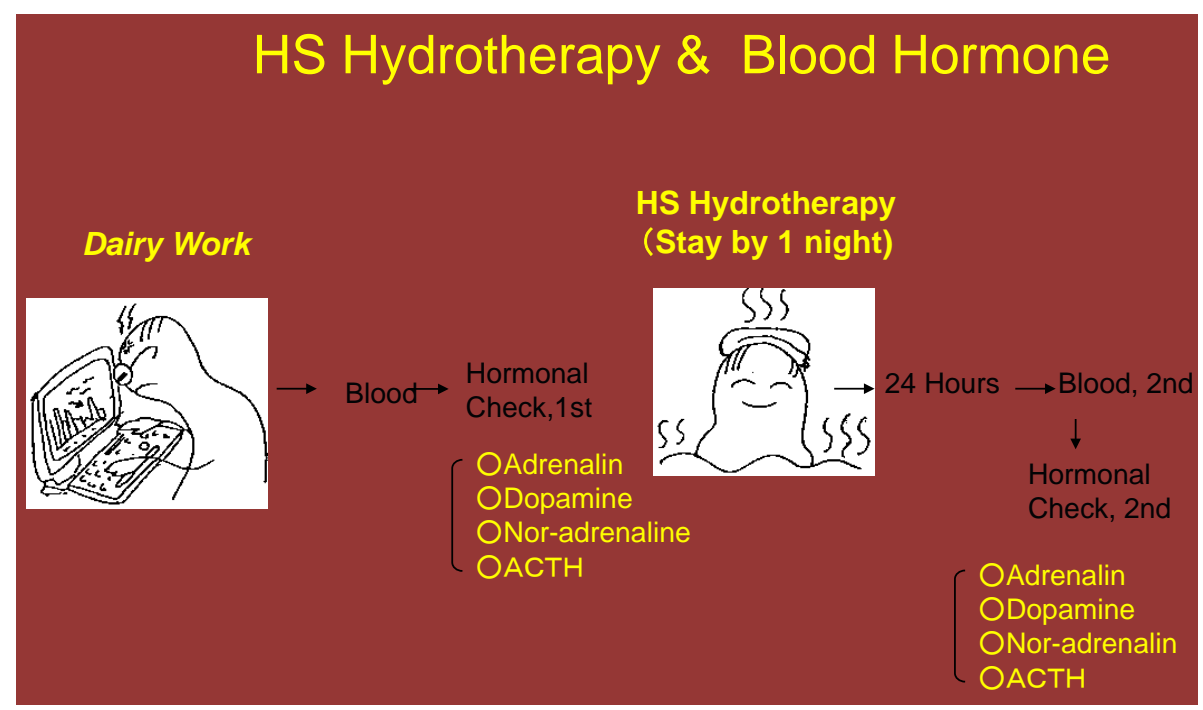

Figure 4. General experimental design to evaluate each CAM therapy. General style for the experiment was designed to collect factors before and after the CAM menu. However, the impotent sings to collect the factor have to keep the time lag is 24 hours in order to avoid the circadian rhythm. Twenty-four hours change of leukocyte counts on the bases of group comparison between pre/post therapy. We sampled peripheral blood from each volunteer before and after CAM therapy, at the same time on each day, in accordance with the consideration of circadian rhythm of leukocyte in this figure, we tried to show the date simply pooled and make mean, then compared.

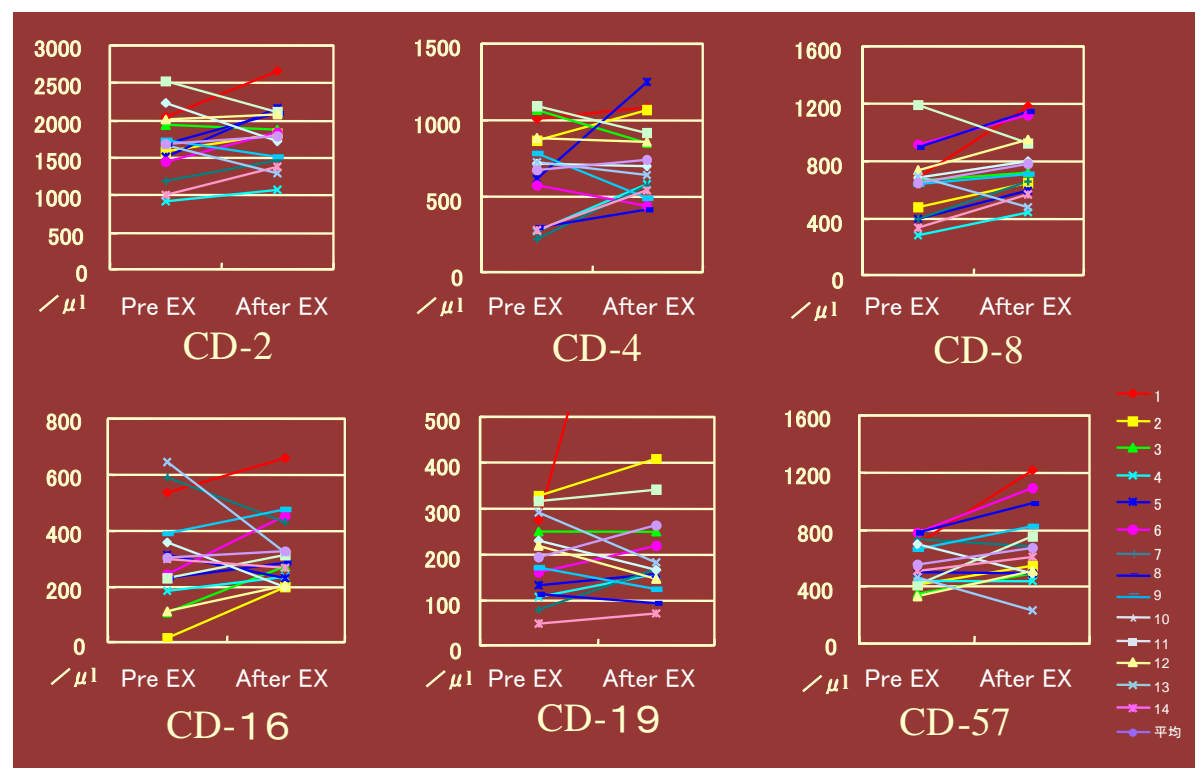

Figure 5. Simple \& conventional presentation of the effect by walking, 4 mets. We first got the data from leukocyte regulation from 24 hours interval. But there were many constitutional difference. For example, up-regulated group, down-regulated and no-changed group. So, in this presentation style, simple sum-up and make mean got the result as no change from this figure.

walking for regulative menu for leukocyte regulation. We had planned two type of impact. One is for waking around $4 \mathrm{~km} /$ hour hour and the other was $8 \mathrm{~km}$ /hour. 8 mets of walking brought ideal regulation, co-efficiency (Figure 9). However, 8 met excursive was not so effective and clearly compared by the value calculated. Moreover, the presentation is one of the scale for appropriate suggestion for someone ask his/her impact of walking. 


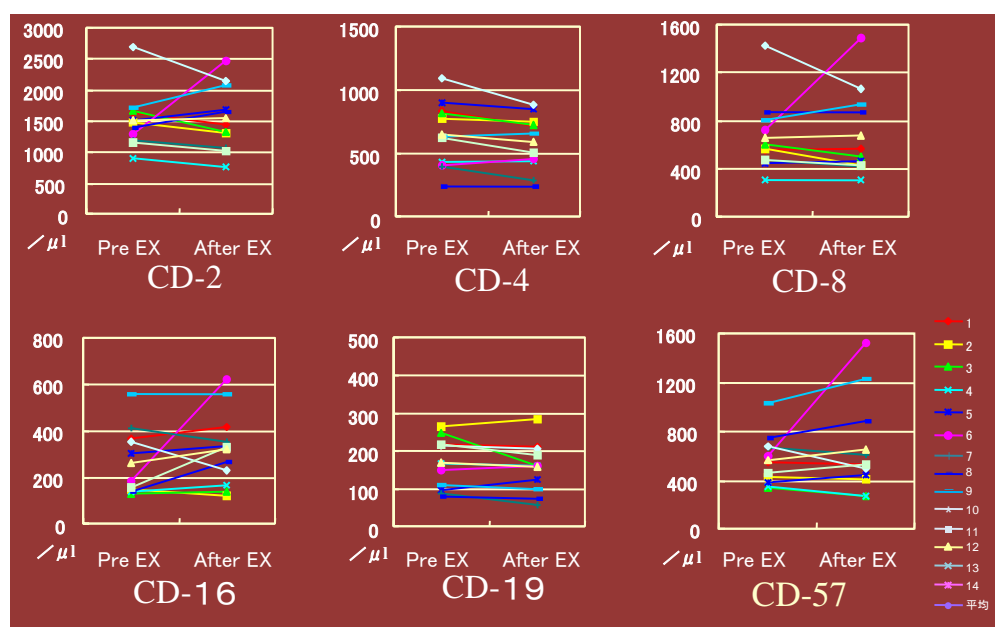

Figure 6. Simple \& conventional presentation of the effect by walking 8 mets. We first got the data from leukocyte regulation from 24 hours interval. But there were many constitutional difference. For example, up-regulated group, down-regulated and no-changed group. So, in this presentation style, simple sum-up and make mean also got the result as no change from this figure.

\section{G TYPE}

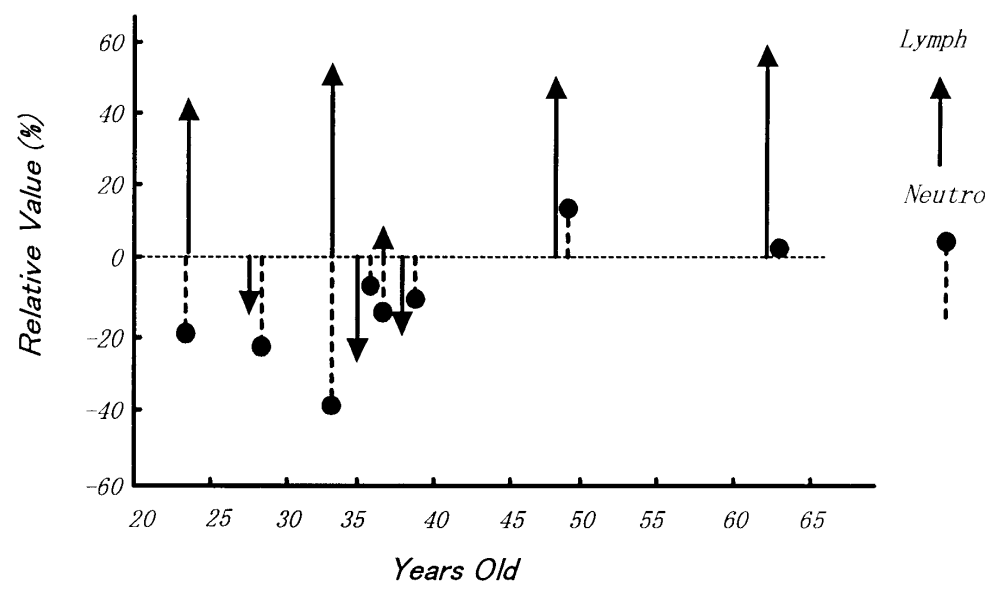

Figure 7. We reported that according to the lymphocyte subset content, lymphocyte rich type showed over $40 \%$ on the other hand granulocyte rich type show over $60 \%$ of granulocyte. Each type exhibited different character even in the same age; sexuality and each age (see Figure 10). In the figure, within the same age and the sex, even in mankind can sorts out as G-rich type (granulocyte 60\%; --- $\rightarrow$ ), and L-rich type (lymphocyte 40\%; ----(11).On the other hand, as a stand point of sex difference, According to this figure, G-rich type changed to increase lymphocyte number, on the other hand, L-rich type increase granulocyte/neutrophyle. It was prominent that the young aged G-type decreased the granulocyte.

For example haw many speed and how many km should do for the best impact? The circumstance, the balneotherapy using the effectiveness of hot-springs hydrotherapy, except for cases of contraindication, has been medically useful approved to be effective in many stress-related disorders and the improvement of dysfunction of the biological rhythm disturbance as well as chronic disease. The mechanism of effects has been reported in many studies, but many things are still unclear. Balneotherapy needs to be treated in general a period of time, but the effectiveness has been suggested even if the short duration hot springs hydrotherapy. We examined the 


\section{TYPE}

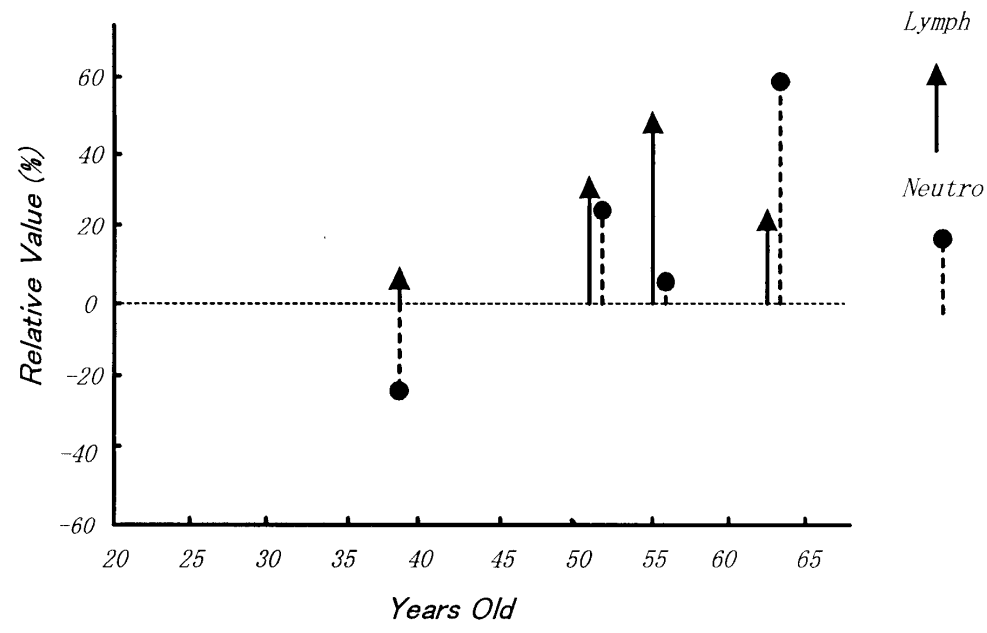

Figure 8. We reported that according to the lymphocyte subset content, lymphocyte rich type showed over $40 \%$ on the other hand granulocyte rich type show over $60 \%$ of granulocyte. Each type exhibited different character even in the same age; sexuality and each age (see Figure 10). In the figure, within the same age and the sex, even in mankind can sorts out as L-rich type (granulocyte 60\%; --- $\rightarrow$ ), and L-rich type (lymphocyte 40\%; ----11). On the other hand, as a stand point of sex difference, According to this figure, G-rich type changed to increase lymphocyte number, on the other hand, L-rich type in crease granulocyte/neutrophyle. It was prominent that the young aged G-type decreased the granulocyte.
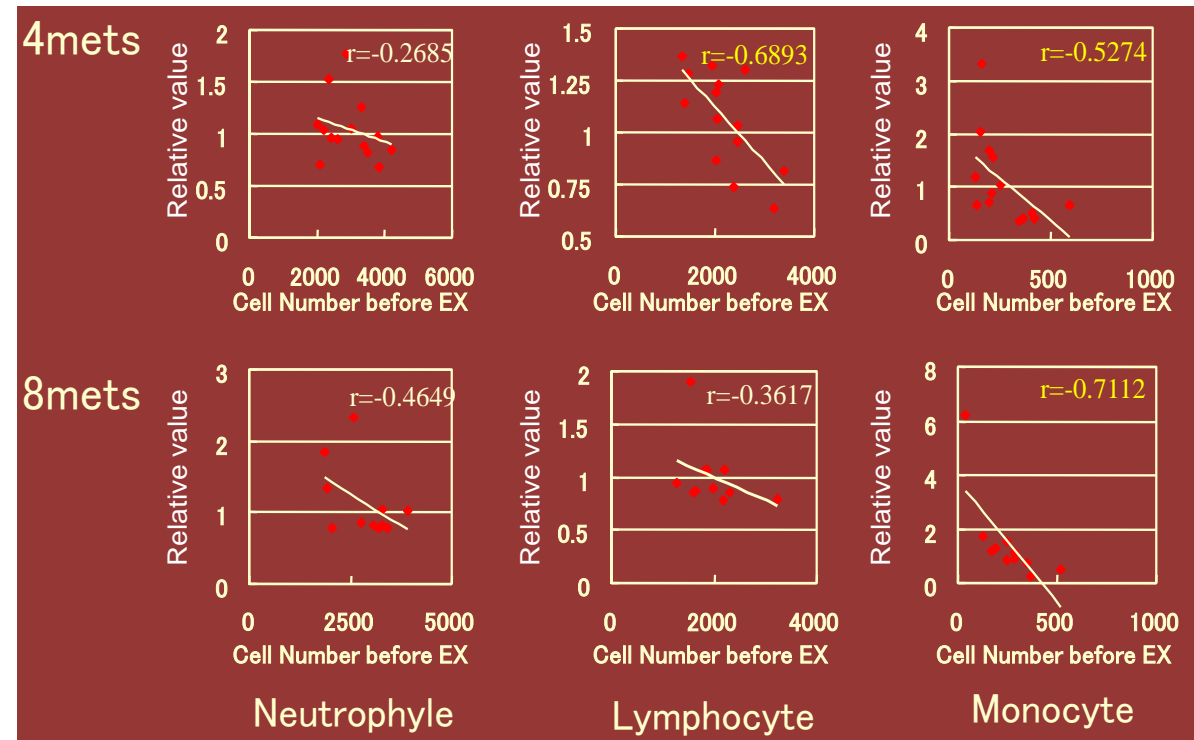

Lymphocyte

Monocyte

Figure 9. The constitution/condition dependent regulation of hot spring hydrotherapy in Lrich type. We reported that according to the lymphocyte subset content, lymphocyte rich type showed over $40 \%$ on the other hand granulocyte rich type show over $60 \%$ of granulocyte. Each type exhibited different character even in the same age, sexuality and each age. In the figure, within the same age and the sex, even in mankind can sorts out as G-rich type (granulocyte 60\%; --- $\rightarrow$ ), and L-rich type (lymphocyte 40\%; ----(11). On the other hand, as a stand point of sex difference, According to this figure, L-rich type increase granulocyte/neutrophyl but decreased lymphocyte percentage. This type was the major group in senile that regulated to increase both lymphocyteand granulocytes. 
effect of hot spring hydrotherapy for a short duration on immune system and reported about the quantitative and qualitative variation of immuno-competent cells [4] [13] [14] [18] [20]. The mechanism is suggested the association with an autonomic nervous system and the endocrine system. Hot spring hydrotherapy for a short duration was expected to stimulate sympathetic nerve or parasympathetic nerve and to change the levels of catecholamines (adrenalin, noradrenaline, and dopamine), which are neurotransmitters and hormones, as well as the number and function of immune cells.

The adrenaline and noradrenaline are secreted by various stress (stimulation); the former from the adrenal medulla and the latter from both the adrenal medulla and the end of sympathetic nerve. They express these functions through the adrenergic receptors (ARs) and regulate the target organ. The adrenergic receptor has two types of $\alpha$ and $\beta$, and there are multiple subtypes $\left(\alpha_{1}, \alpha_{2}, \beta_{1}, \beta_{2}, \beta_{3}\right)$ [21]. These subtypes present the vascular $\left(\alpha_{1}\right)$, the presynaptic terminal $\left(\alpha_{2}\right)$, the heart $\left(\beta_{1}\right)$, the bronchial muscle of the lung $\left(\beta_{2}\right)$, fat cells $\left(\beta_{3}\right)$, respectively. The $\alpha$-receptor stimulation causes Broncho-dilatation and vasodilatation, the $\beta 1$-receptor stimulation causes an increase in heart rate and lipolysis, and the $\beta_{2}$-receptor stimulation causes bronchoconstriction, vasodilatation, and muscle glycogen resolution. The adrenaline provides heart activation by effect on $\alpha$ - and $\beta$-receptors, and the noradrenaline provides blood pressure rises by strongly effect on $\alpha$-receptors. In addition to the above, it has been reported that the ARs are present on leucocyte membranes [21]-[24] and that the level of expression of $\beta$-ARs in lymphocytes was examined by radio ligand (125I-iodopindolol) binding, but the details of subtype of $\beta$-receptors have not been disclosed. The subtypes of the AR are able to be analyzed by flow cytometry (FCM) method. In this study, we described how hot spring hydrotherapy influences leukocyte, lymphocyte subpopulations expressing $\beta_{2}$-AR and the levels of catecholamine in human peripheral blood.

Abo reported that according to the lymphocyte subset content, lymphocyte rich type showed over $40 \%$ on the other hand granulocyte rich type show over $60 \%$ of granulocyte. Each type exhibited different character even in the same age, sexuality and each age.

In the Figures, within the same age and the sex, even in mankind can sorts out as G-rich type (granulocyte $60 \%$ ), and L-rich type (lymphocyte 40\%).

On the other hand, as a standpoint of sex difference, the ladies belong to L-rich type but the man belongs Grich type. According to the age-related change, G-rich type of man change to L-rich type within the same sex (Figure 10).

\section{Evidence-Based Expression of Constitution, SCS; Super Constitution System}

Abo reported that the ratio of granulocyte and lymphocyte are different in each individual. This difference reveals the sexual different (Figure 10). Moreover, both leucocyte are daily regulated by circadian rhythm and the environmental factors [23] [24]. But each cycle of leukocyte, granulocyte and lymphocyte are different each other. So the experimental design is necessary to synchronized with the time to check, for example, the number of leukocyte from peripheral blood. Hot Spring Hydro Therapy Regulate Peripheral Leukocyte Together with Emotional Hormone and Receptor Positive Lymphocytes According to Each Constitution/Condition is important to conscious of circadian rhythm. Abo reported that it was possible to sort the constitution, granulocyte-rich individual and lymphocyte-rich one with the peripheral leukocyte [25] [26].

Within a same individual, granulocyte increase in the daytime, on the other hand, lymphocyte increased in the nighttime in a cycle $24 \mathrm{hrs}$. So we have to compare the effect of each menu for the peripheral leukocyte on the same time before and after the menu.

It was reported that the leukocyte subset, granulocyte and lymphocyte regulated by various factors. One major point is that they are regulated by autonomous nervous system, resulting in circadian rhythm [23].

Therefore, in order to access the effect within a short time, it is necessary to consider this factor to adjust the time to collect the sample. For example, efficacy and impact of walking exercise has been widely recognized. However, the majority of walkers did not have a scientific background to know the best exercise menu for the one' QOL; quality of life. The purpose of this study was to demonstrate the best menu of walking that regulates the peripheral white blood cells in number and function as a marker of QOL expression Figure 9 and Figure 10.

\section{Granulocyte/Lymphocyte Ratio Reveals Constitution}

Traditionally, each heat therapy has its own character and efficacy for various complaints. Through the years, each water source was evaluated for its specific properties and with the advent of better transportation in our mountainous land, even remote springs in the mountains were visited for their specific medicinal effect. For 


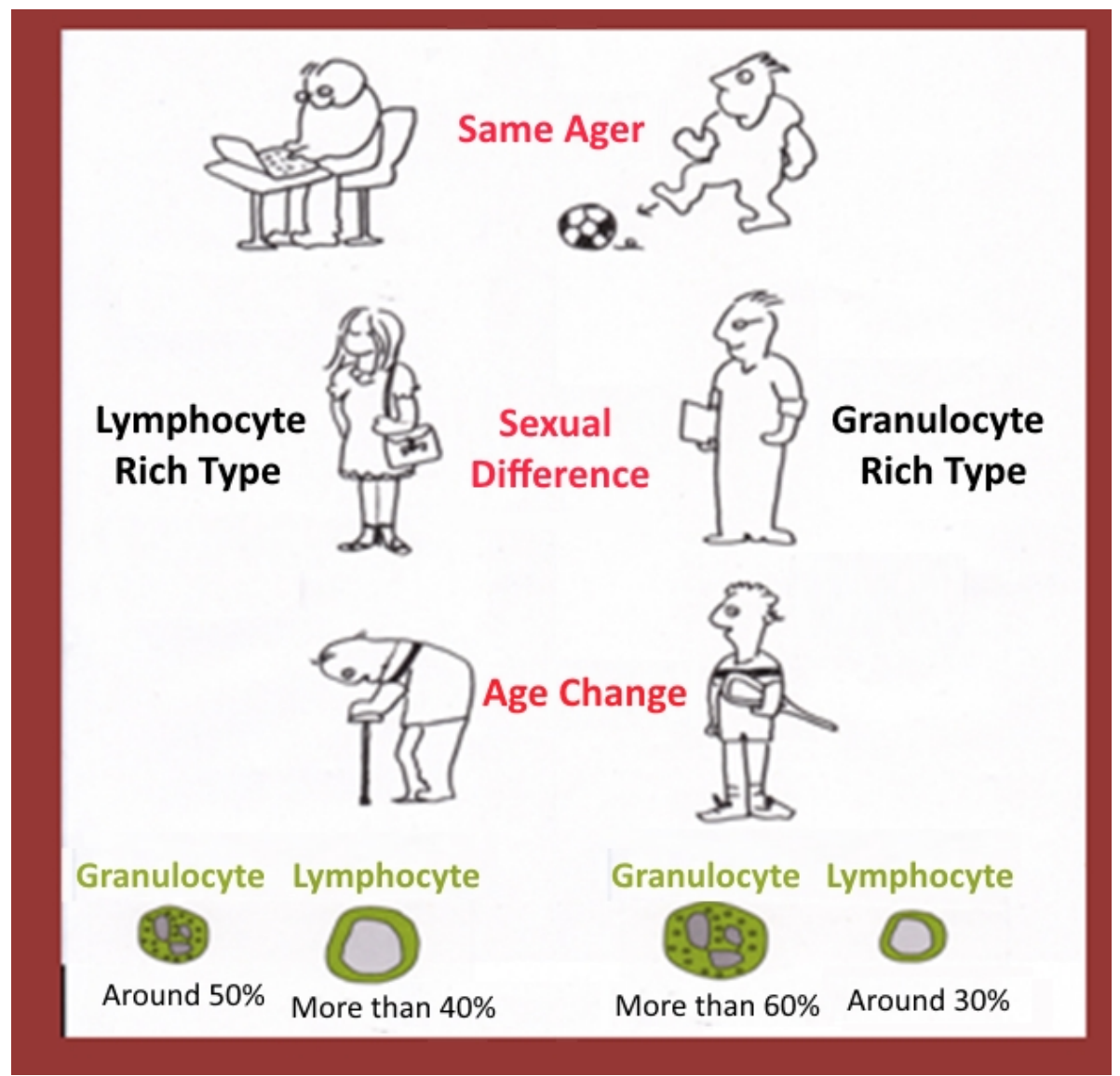

Figure 10. An ontogenical and phyogenetical consideration of constitution, for the best condition and the best QOL. In the figure, within the same age and the sex, even in mankind can sorts out as G-rich type (granulocyte 60\%), and L-rich type (lymphocyte 40\%). On the other hand, as a stand point of sex difference, the ladies belong to L-rich type but the man belongs G-rich type. According to the age-related change, G-rich type of man change to L-rich type within the same sex.

example, the hot spring of Yuwaku-Onsenn, which is located just below our research facility, was known all over the area to cure hemorrhoids. However, almost all the judgments of efficacy are VAS (visual analog scale). The proprietors of the hot spring inns say that many of the guests came to cure that sort of ailment since about 20 years ago. Now in Japan, hot spring hydrotherapy is often used as a supplementary therapy for many diseases [21] [28] [29]. It has shown to reduce surgical complaints such as shoulder pain, amyloidosis and various rheumatic problems. It can also lighten the burden to the heart and improve the condition of patients who suffer from emphysema as well as other respiratory ailments. Hot springs have also traditionally functioned as places to relax and enjoy oneself, even though resort-type hot springs always exist along with those for illnesses. In recent years, trends have been seen that even remote hot springs, such as the one below our facility, transform into fashionable resorts for the healthy to visit for relaxation and stress release. It is interesting to note that this historical duplicity encompassed by hot springs has also entered the world.

\section{An Importance to Regulate Neutral in Both Leucocyte in Number}

Each volunteer was prepared their blood before start to visit hot spring village after informed consented to the experimental purpose by written Ethics of the Committee in Kanazawa Medical University. After lodging the hotel, volunteers enjoyed Japanese type hot spring bath system, with no under wear in the bath room/pool separately prepared for ladies and gentleman. We suggested taking a bath totally 2 - 3 times within two days for the aim of trial. Beside the advance of VAS: visual analog scale in our investigation, we measured the total number 
of leukocyte and two major subsets, granulocyte and lymphocytes regulated before and after hot spring hydrotherapy.

We tried to express the effect of peripheral total leukocyte number by individual level of change and plot in the $\mathrm{x}$-axis as in each age. As was in Figure 11, the groups was separated in to two, up-regulated individuals and down-regulated one. The correlation of change was expressed as a linear function. Figure 11 was ideal change of effect with hot spring hydrotherapy, showing the best inclination. The results showed that these subsets could reflect the number and function of immuno-competent cells. For example, in an individual with a low granulocyte number, the number increased after treatment, while it decreased in another individual with a higher cell number (Figure 12).

We sampled peripheral blood from the 12 volunteers before and after hot sand/hydro therapy, at the same O'clock on the day, with the respect of circadian rhythm [25] [27] of leukocyte. These subjects participated in this study after giving their informed consent. We conducted the experiment at Yuwaku Onsen Spa (Kanazawa, Ishikawa Pref., Japan) in one night of the day. The spring quality is a weak sodium chloride with sodium carbohydrate of the water temperature $41^{\circ} \mathrm{C} \pm 1{ }^{\circ} \mathrm{C}$. During the night and in the morning of the next day, they had a bath in the hot spring two or three times, for 20 - 30 minutes each time. Hot sand spa had performed and bathing for 40 mins. Time interval of blood sampling between before and after hot-spring hydrotherapy was approximately 24 hours. Measurements of the total and differential leukocyte counts and 3 catecholamines levels in the peripheral blood.

We ordered on the laboratory of Ishikawa Prefecture Preventive Medicine Association about the total and differential leukocyte counts and the levels of 3 catecholamines (adrenaline, noradrenaline, and dopamine) in the peripheral blood from the subjects. The total and differential leukocyte counts were measured by the automated hematology analyzer XE-2100 (Sysmex, Inc., Kobe, Japan). The levels of catecholamines were measured by high performance liquid chromatography (HPLC) system (Tosoh Co. and Hitachi High-Technologies Co., ToKyo) The immune system is a totally integrative system. It includes brain, endocrine and immune system. For example, an immune system contains various cells, tissues and organs that protect organisms against potentially

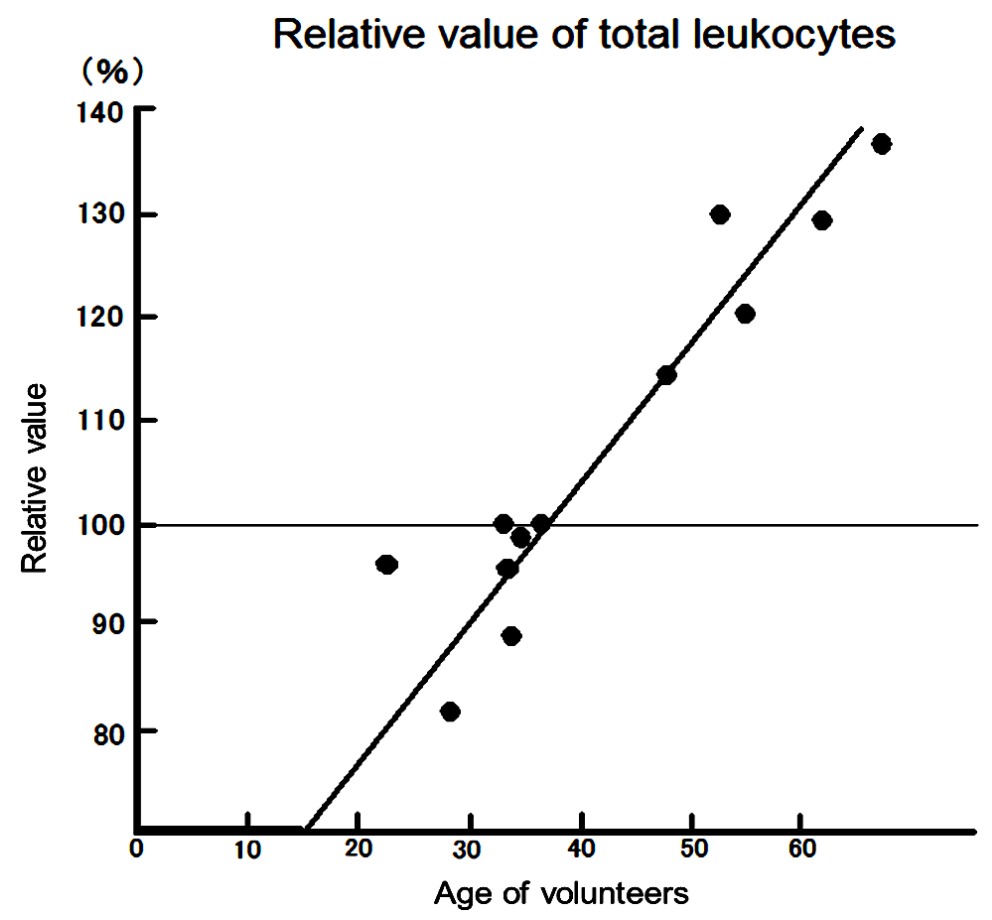

Figure 11. The constitution/condition dependent regulation and presentation by linear slant and judging by their tangent value/ co-efficiency. In the figure, the trial was made to show the each slant as a representative of the regulational effect to CD positive lymphocyte. The figure shows after light excursive/walking 4 mets and 8 mets. 


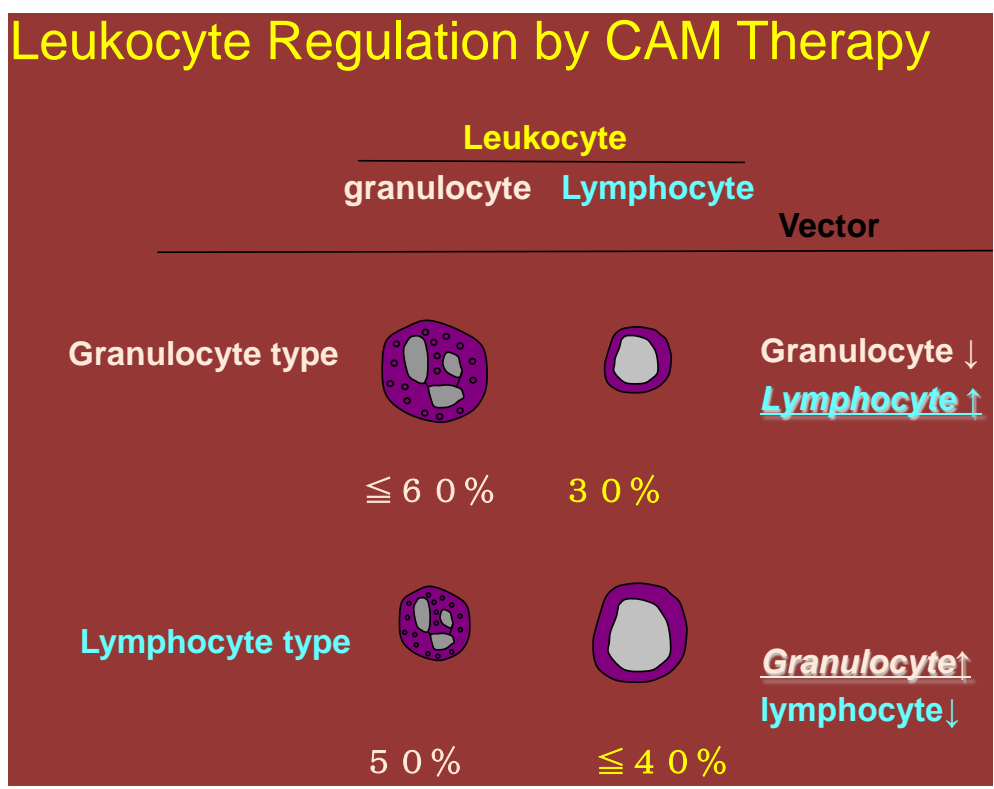

Figure 12. A graphical presentation for the summary of the hot spring hydrotherapy. We tried to express the effect of peripheral total leukocyte number by individual level of change and plot in the $\mathrm{X}$-axis as in each age. Variations in leukocyte subpopulations in the peripheral blood before and after each CAM therapy.

harmful pathogens from the external environment. The present concept of the word immunity has proposed considerably from its original definition to the standard scale for CAM (complementary and alternative medicine). Quite literally, its earlier usage referred to exemption from military service or the paying of taxes. Now, immunity not only "frees" one from disease but also the standard scale for CAM (Figure 3, Figure 10, Figure 12).

\section{Constitution Dependent Regulations Are Important for QOL}

The recent excessive commercialization is particularly confusing for patients and doctors who seek remedies for heretofore undefined symptoms. Furthermore, since these treatments have not undergone strict testing, they are not always safe and the same drug may have different effects according to the individual patient and dosage. Complicated considerations are necessary for the application of practices such as those found in Chinese traditional medicine.

Recently, alternative and complementary medicines together with oriental and traditional medicines have attracted much attention. This new interest includes aromatherapy and herbal medications, acupuncture, moxibustion and yoga.

However, these therapies have not been well defined. Some are simply based on legend or belief while others are traditionally applied but without scientific evidence. Then the assessment of each therapy and remedy need for their capacity through a scientific methods especially developed by Western Medicine. Although the word Alternative \& Complementary Medicine is not popular enough in Japan than US and European countries, because in oriental countries so called Alternative and Complementary Medicine in Europe and north America is authorized medicine but not alternative one in the long histories in the world. Here I would like to introduce you where the alternative and complementary medicine now in Japan as well as in each country in the world and where should it be going in the future.

Each volunteer was prepared their blood before start to visit hot spring village after informed consented to the experimental purpose by written Ethics of the Committee in Kanazawa Medical University. After lodging the hotel, volunteers enjoyed Japanese type hot spring bath system, with no under wear in the bath room/pool separately prepared for ladies and gentleman. We suggested taking a bath totally $2-3$ times within two days for the aim of trial. Beside the advance of VAS: visual analog scale in our investigation, we measured the total number of leukocyte and two major subsets, granulocyte and lymphocytes regulated before and after hot spring hydro- 
therapy.

We tried to express the effect of peripheral total leukocyte number by individual level of change and plot in the $\mathrm{x}$-axis as in each age. As was in Figure 11, the groups was separated in to two, up-regulated individuals and down-regulated one. The correlation of change was expressed as a linear function. Figure 11 was ideal change of effect with hot spring hydrotherapy, showing the best inclination. The results showed that these subsets could reflect the number and function of immuno-competent cells. For example, in an individual with a low granulocyte number, the number increased after treatment, while it decreased in another individual with a higher cell number (Figure 7, Figure 8).

We sampled peripheral blood from the 12 volunteers before and after hot sand/hydro therapy, at the same O'clock on the day, with the respect of circadian rhythm [25] [27] of leukocyte. These subjects participated in this study after giving their informed consent. We conducted the experiment at Yuwaku Onsen Spa (Kanazawa, Ishikawa Pref., Japan) in one night of the day. The spring quality is a weak sodium chloride with sodium carbohydrate of the water temperature $41^{\circ} \mathrm{C} \pm 1{ }^{\circ} \mathrm{C}$. During the night and in the morning of the next day, they had a bath in the hot spring two or three times, for 20 - 30 minutes each time. Hot sand spa had performed and bathing for 40 mins. Time interval of blood sampling between before and after hot spring hydrotherapy was approximately 24 hours. Measurements of the total and differential leukocyte counts and 3 catecholamines levels in the peripheral blood.

We ordered on the laboratory of Ishikawa Prefecture Preventive Medicine Association about the total and differential leukocyte counts and the levels of 3 catecholamines (adrenaline, noradrenaline, and dopamine) in the peripheral blood from the subjects. The total and differential leukocyte counts were measured by the automated hematology analyzer XE-2100 (Sysmex, Inc., Kobe, Japan). The levels of catecholamines were measured by high performance liquid chromatography (HPLC) system (Tosoh Co. and Hitachi High-Technologies Co.).

As we here took an overview about the today's QOL assessment for the medicine in the east and the west, I think it is high time this medicine should be standardized uniformly and Japan could play an important roll in this task.

In Japan, the Eastern Medicine especially the herbal medicine was once the central medical care for long time until the Meiji Government decided to import the Western medicine as the ordinary medical care. Thus in Japan not only the Western but also the Eastern medicine could have been developed, and Japan now takes great pride in the longevity. This is why I suppose Japan should take the initiative for the standardization of the alternative and complementary medicine.

The problem is how it should be standardized. Here I would like you to propose something. Most of the alternative medicine works through affecting the regularly system inside the body. For example, recent studies revealed the herbal medicine caused the interaction between the immune system, the endocrine system and the nervous system. Therefore observing the immune system by like sampling the peripheral blood might be an indicator for the standardization of the alternative medicine. Due to the above difficulties, this realm of medicine has often been shut out of the serious journals of western medicine. A new Journal concerning around complementary, alternative and traditional medicine" will be launched in a desire to ameliorate this situation, by encouraging the publication of original scientific papers based on sound scientific guidelines, but without prejudice against the possible efficacy of these new and ancient treatments (Figure 3).

\section{Leucocytes Subsets Regulated in the Number and Function}

The immune system shares with the nervous system at least two characteristics. The young individual is born with a certain potential to learn and to react to numerous and varied environmental stimuli both systems can lean. Once information is learned by the immune and nervous systems, it becomes in a sense imprinted, and each system retains the information in varying degrees, a process defined as memory. Despite the intense learning that young systems must do subsequent to birth and will continue to do throughout their life time, infants are born into the world with certain innate behavior patterns controlled by the nervous system, and certain innate or characteristic natural immunities. Innate or natural, immunity includes all non-specific resistance or specific immune responses.

Nonspecific reaction of the PE fluorescence was found in the isotype control. Therefore, the real values of the AR expressing cell counts were calculated by subtracting control values. The CD19 ${ }^{+}$cells were observed nonspecific reaction which seems to response of the second antibody. However, the significant variation was not 
seen from the comparison of $\beta_{2}$-AR expressing cells before and after hot-spring hydrotherapy. The mean $\%$ of $\beta_{2}$-AR expressing cells in the lymphocyte subsets was $18-19 \%$ in $\mathrm{CD}^{+}$cells, $5 \%$ in CD4 ${ }^{+}$cells, $57 \%-63 \%$ in $\mathrm{CD}^{+}$cells, and $93 \%-95 \%$ in CD56 ${ }^{+}$cells. That in CD19 ${ }^{+}$cells was approximately $100 \%$ (data not shown), but we were not able to be confirmed because it was very likely to be the nonspecific reaction. The adrenaline and noradrenaline are secreted by various stress (stimulation); the former from the adrenal medulla and the latter from both the adrenal medulla and the end of sympathetic nerve. They express these functions through the adrenergic receptors (ARs) and regulate the target organ. The adrenergic receptor has two types of $\alpha$ and $\beta$, and there are multiple subtypes $\left(\alpha_{1}, \alpha_{2}, \beta_{1}, \beta_{2}, \beta_{3}\right)$ [4]. These subtypes present the vascular $\left(\alpha_{1}\right)$, the pre- synaptic terminal $\left(\alpha_{2}\right)$, the heart $\left(\beta_{1}\right)$, the bronchial muscle of the lung $\left(\beta_{2}\right)$, fat cells $\left(\beta_{3}\right)$, respectively. The $\alpha$-receptor stimulation causes bronchodilatation and vasodilatation, the $\beta 1$-receptor stimulation causes an increase in heart rate and lipolysis, and the $\beta_{2}$-receptor stimulation causes bronchoconstriction, vasodilatation, and muscle glycogen resolution. The adrenaline provides heart activation by effect on $\alpha$ - and $\beta$-receptors, and the noradrenaline provides blood pressure rises by strongly effect on $\alpha$-receptors. In addition to the above, it has been reported that the ARs are present on leucocyte membranes [20] [21] and that the level of expression of $\beta$-ARs in lymphocytes was examined by radioligand (125I-iodopindolol) binding, but the details of subtype of $\beta$-receptors have not been disclosed. The subtypes of the AR are able to be analyzed by flow cytometry (FCM) method. In this study, we described how hot spring hydrotherapy influences leukocyte, lymphocyte subpopulations expressing $\beta_{2}$-AR and the levels of catecholamine in human peripheral blood.

The recent excessive commercialization is particularly confusing for patients and doctors who seek remedies for heretofore undefined symptoms. Furthermore, since these treatments have not undergone strict testing, they are not always safe and the same drug may have different effects according to the individual patient and dosage. Complicated considerations are necessary for the application of practices such as those found in Chinese traditional medicine. Recently, alternative and complementary medicines together with oriental and traditional medicines have attracted much attention. This new interest includes aromatherapy and herbal medications, acupuncture, moxibustion and yoga. However, these therapies have not been well defined. Some are simply based on legend or belief while others are traditionally applied but without scientific evidence. Then the assessment of each therapy and remedy need for their capacity through a scientific methods especially developed by Western Medicine. Although the world Alternative \& Complementary Medicine is not popular enough in Japan than US and European countries, because in oriental countries so called Alternative and Complementary Medicine in Europe and north America is authorized medicine but not alternative one in the long histories in the world. Here I would like to introduce you where the alternative and complementary medicine now in Japan as well as in each country in the world are and where should it be going in the future (Figure 3).

\section{The Impact of Regulation Can Makes Linear Slant, SCS}

The immune system is a totally integrative system. It includes brain, endocrine and immune system. For example, an immune system contains various cells, tissues and organs that protect organisms against potentially harmful pathogens from the external environment. The present concept of the word immunity has proposed considerably from its original definition to the standard scale for CAM (complementary and alternative medicine). Quite literally, its earlier usage referred to exemption from military service or the paying of taxes. Now, immunity not only "frees" one from disease but also the standard scale for CAM.

The immune system shares with the nervous system at least two characteristics. The young individual is born with a certain potential to learn and to react to numerous and varied environmental stimuli both systems can lean. Once information is learned by the immune and nervous systems, it becomes in a sense imprinted, and each system retains the information in varying degrees, a process defined as memory. Despite the intense learning that young systems must do subsequent to birth and will continue to do throughout their life time, infants are born into the world with certain innate behaviour patterns controlled by the nervous system, and certain innate or characteristic natural immunities. Innate or natural, immunity includes all non-specific resistance or immune mechanisms, whereas specific active acquired immunity is immunity deliberately induced by immunization (e.g. immunity to bacteria and virus).

Man, other complex vertebrates, and even many invertebrates have evolved a system of internal transport for communicating components of the immune system. It is the blood, within the circulatory system, that executes these tasks. The blood contains two major types of cells: erythrocytes, or red blood cells, and leukocytes, or 
white blood cells. Leukocytes play important roles in the immune system. There are two basic types of leukocytes: the non-granular and the granular. The non-granular (agranular) leukocytes are further divided into two types: lymphocytes and monocytes.

Lymphocytes possess antibody receptors for antigens on their surfaces, and are thus vital to specific immune response throughout the entire body, where they freely move about. Monocytes are produced in the bone marrow, but like other blood cells they are eventually found in the blood. They frequently exhibit amoeboid movement, and they are voracious phagocytes when they enter connective tissues as macrophages from the blood. Both are important for quick, on the spot, phagocytosis (Figure 12).

\section{Factors Affect the Lymphocyte Ratio}

\subsection{Total and Differential Leukocyte Counts}

The total and differential leukocyte counts in the peripheral blood of 9 subjects tended to decrease after hot spring hydrotherapy and the granulocyte counts significantly decreased $(p<0.05)$ judging from simple sum up and mean (Figure 5, Figure 6, Figure 12).

\subsection{Catecholamines Levels in the Peripheral Blood}

The adrenaline levels significantly decreased after hot spring hydrotherapy $(p<0.05)$; the noradrenaline and dopamine levels ten to decrease (Figure 13). The constitution dependent analysis, the detail change and vector

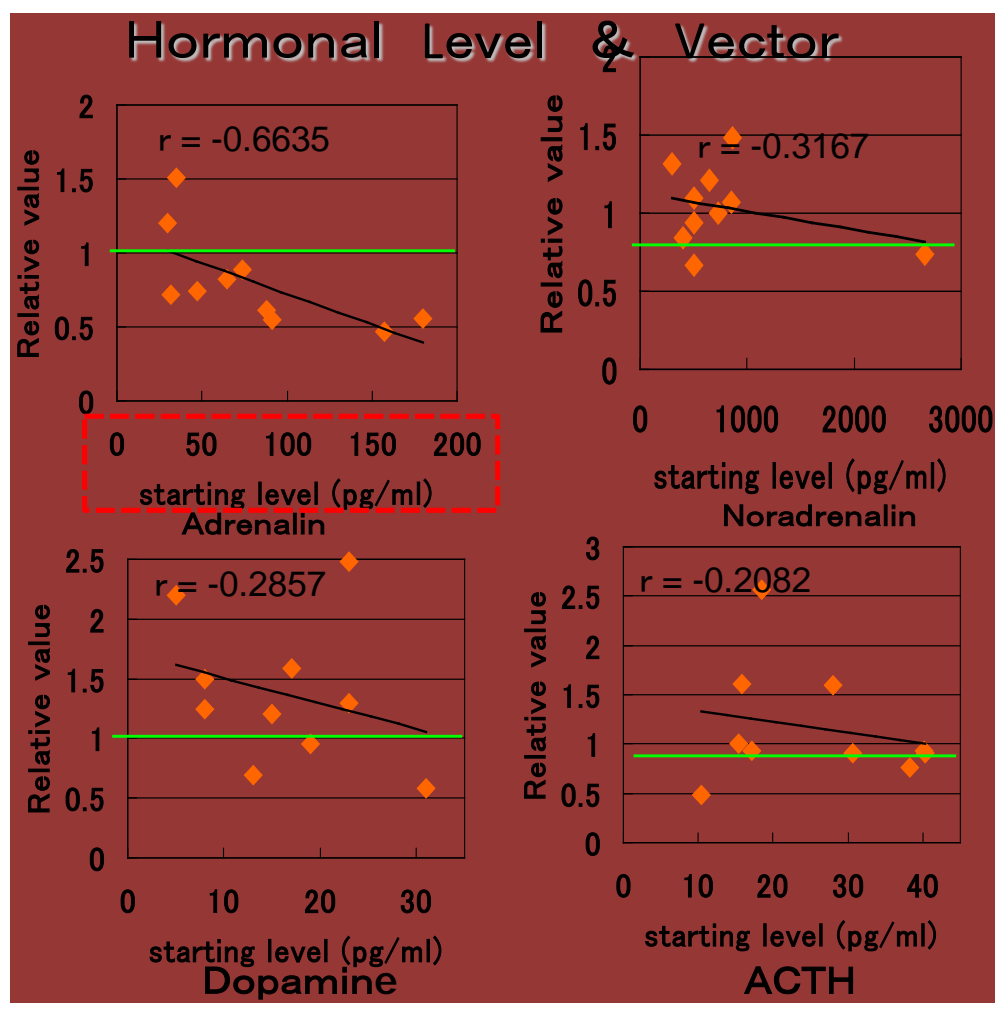

Figure 13. A emotional hormone also regulated by CAM therapy. The constitution dependent analysis for (adrenaline and dopamine). Each spot were obtained from the calculation comparing relative value from before and after levels in the serum, catecholamines levels in the peripheral blood within the figure, adrenalin and dopamine were regulated as was the same in leukocyte regulation. The clear slant was made from the data especially, in regulation in adrenalin, overall trend was decrease but was dose dependent manner of the value the day before. Also, dopamine was increased but also dose dependent manner as slant making. 
of each change could find from individual data, showing higher value volunteer down regulated much more than lower leveled one. The results indicate that the hot-spring hydrotherapy may influence the secretion of adrenaline from the adrenal medulla.

\subsection{FCM Analysis of $\beta_{2}$-AR Expresszion in Lymphocyte Subsets}

The analysis of $\beta_{2}$-AR expressing cells and CD positive cells by FCM was measured by gating in the lymphocytes region on the scattered gram. Nonspecific reaction of the PE fluorescence was found in the isotype control. Therefore, the real values of the AR expressing cell counts were calculated by subtracting the control values. The $\mathrm{CD}_{19^{+}}$cells were observed nonspecific reaction which seems to response of the second antibody. The comparison of each lymphocyte subpopulations before and after hot spring hydrotherapy showed that the CD8 ${ }^{+}$ cell and $\mathrm{CD}_{56}{ }^{+}$cell counts tended to increase (Figure 14). However, the significant variation was not seen from the comparison of $\beta_{2}$-AR expressing cells before and after hot-spring hydrotherapy. The mean \% of $\beta_{2}$-AR expressing cells in the lymphocyte subsets was $18 \%-19 \%$ in $\mathrm{CD}^{+}$cells, $5 \%$ in $\mathrm{CD}^{+}$cells, $57 \%-63 \%$ in $\mathrm{CD}^{+}$ cells, and 93\% - 95\% in CD56 ${ }^{+}$cells. That in CD19+ cells was approximately $100 \%$ (data not shown), but we were not able to be confirmed because it was very likely to be the nonspecific reaction.

We examined the correlation with the rate of change in adrenaline levels and the rate of change in $\beta_{2}$-AR expressing cell counts of each subset or that in each CD-positive cell counts before and after hot-spring hydrotherapy. In the CD-positive cells, the rate of change in adrenaline levels was a positive correlation with that in the levels of $\mathrm{CD} 56^{+}$cells, $\mathrm{CD} 8^{+}$cells, and $\mathrm{CD}^{+}$cells; in particular, a correlation with $\mathrm{CD}^{+} 6^{+}$and $\mathrm{CD}^{+}$cells was high. In $\beta_{2}$-AR expressing cells, the rate of change in adrenaline levels was a positive correlation with the rate of change in the levels of $\beta_{2}-\mathrm{AR}^{+} \mathrm{CD}_{5}{ }^{+}$cells. These results suggested that the variation in adrenaline levels is correlated with $\mathrm{CD}_{56}{ }^{+}$cells.

Each volunteer was prepared their blood before start for trial and after informed consented to the experimental purpose by written Ethics of the Committee in Kanazawa Medical University. We tried to exhibit the effect of peripheral total leukocyte number by individual level of change and plot in the x-axis as in each value before the exercise. As was in Figure 9, the relative value (\%) was calculated before and after the exercise and plotted in figure of the $\mathrm{X}$-axis according to the value before exercise. As a result of the trial, there found three groups,

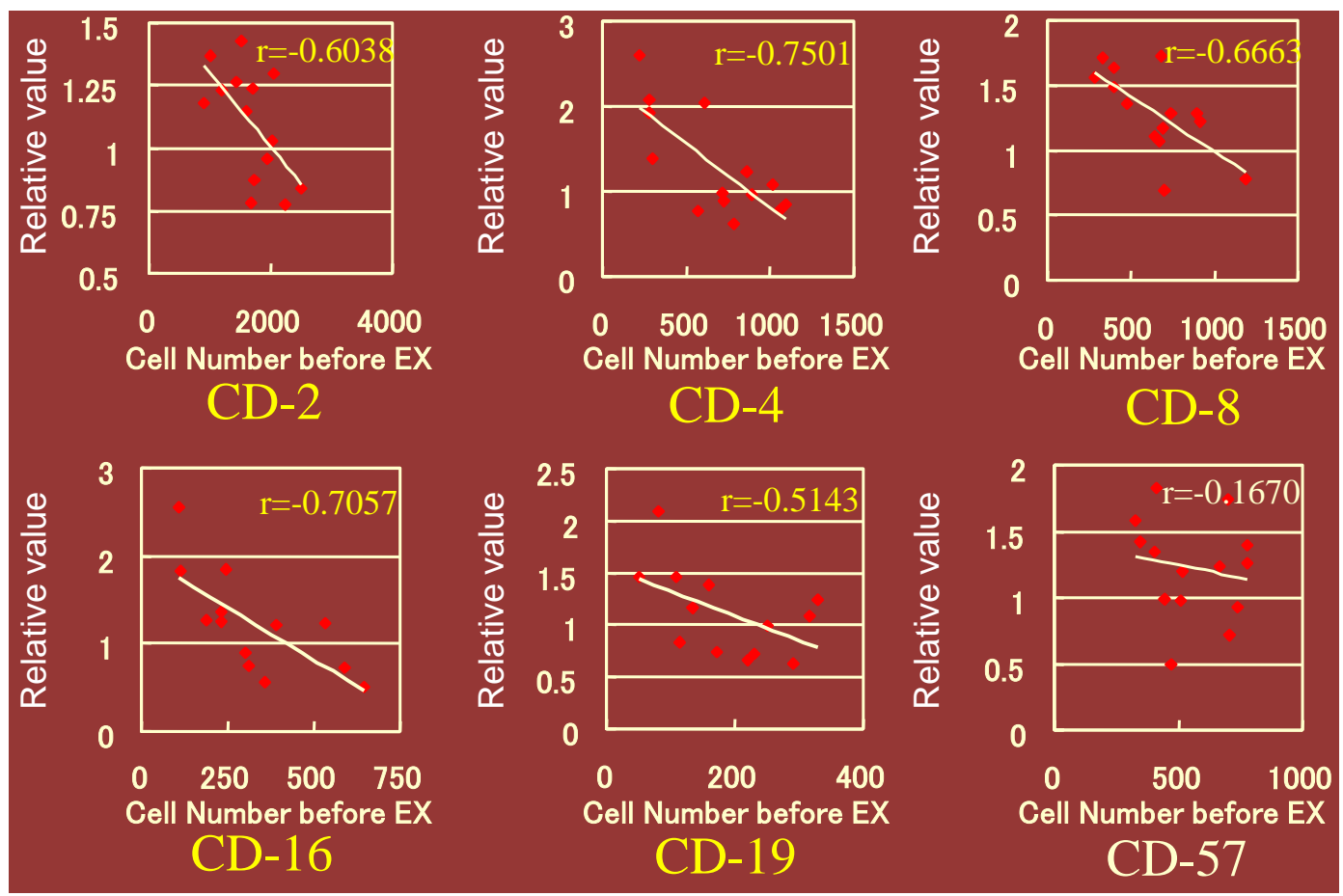

Figure 14. In the figure, the trial was made to show the each slant as an representatives of the regulational effect to CD positive lymphocyte. The figure show after hot spring hydro therapy. 
separated, up-regulated individuals and down-regulated one and was no change. The correlation of change was expressed as a linear function and significant reverse correlation, -0.6893 indicating ideal value of correlative index -0.5 (Figure 14). The data obtained from 4mets exercise was brought ideal regulation. However, 8 mets could not bring such a effect significantly. About the leukocyte subset, the ideal regulation of lymphocyte and monocyte were found by floor heating room.

\subsection{Twenty-Four Hours Change of Leukocyte Counts after Heating by Hot Air from Overhead}

None of volunteer was dropped out with any serious problem. In trial, we set up room temperature also 18C at the $150 \mathrm{~cm}$ in high from floor, we tried to express the effect of peripheral total leukocyte number by individual level of change and plot in the X-axis as in each value before the exercise as is in 3.1. As shown in lower panel of Figure 15, the correlation was exhibited on a linear function but good correlation was retreated from the results the data obtained from 8 mets exercise was lesser regulatory effect than that compared by 4mets of exercise. About the leukocyte subset, the regulation by lymphocytes were more remarkable. However, only the monocyte exhibited significant change by both.

\subsection{Leukocyte Subsets Regulation by Floor Heating}

After one-weeks of working in the conditioned room as HCFH or HHFC, we set up the different mode of trial at the same course and by the same volunteers. None of volunteer was dropped out with any serious problem. In this time, we set up HCFH system, asking to work about for 5 days. We selected four hormones and accessed the effect of HCFH or HHFC conditioned room. After working about regular desk working in the HCFH conditioned room, each volunteer was down regulated the adrenaline, cortisone and nor-adrenalin. However, dopamine level was up-regulated for all the individuals tested. On the other hand, HHFC system showed reversed effect for all the hormones in this text (Figure 16).

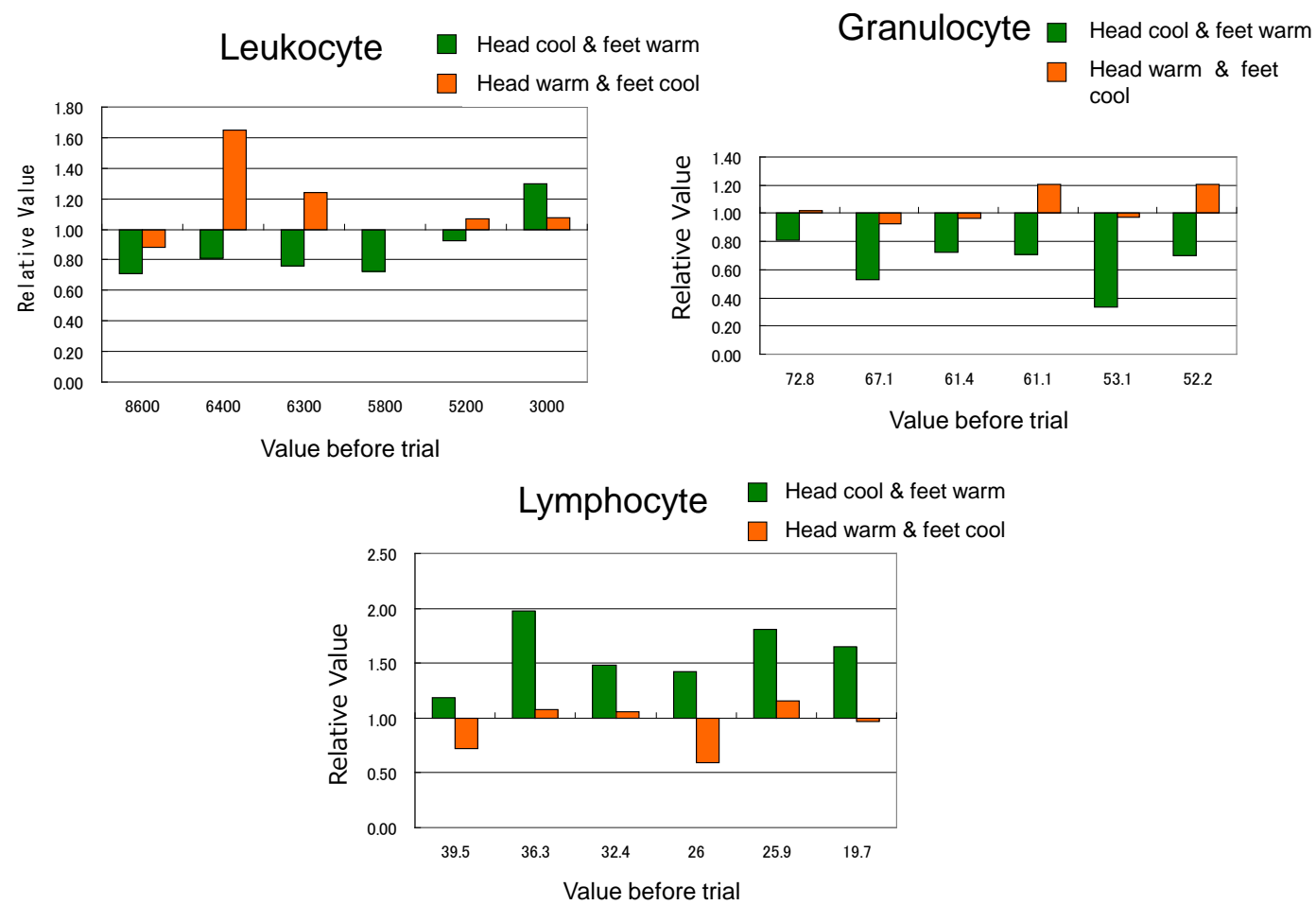

Figure 15. We tried to express the effect of peripheral total leukocyte number by individual level. The results were plotted in the $\mathrm{x}$-axis as in each value before the trial. The relative value (\%) of post trial was calculated with before and after and plotted in the Y-axis. Total leukocytes, Leukocyte subset, granulocyte and lymphocyte were also traced in the Figures. 
Head cool \& feet warm

Granulocyte

Head cool \& feet warm

Head warm \& feet cool

Head warm \& feet cool

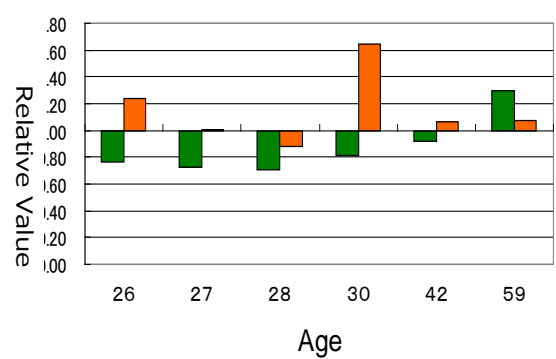

Lymphocyte

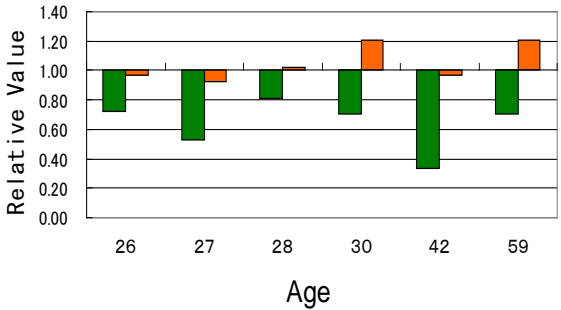

Head cool \& feet warm

Head warm \& feet cool

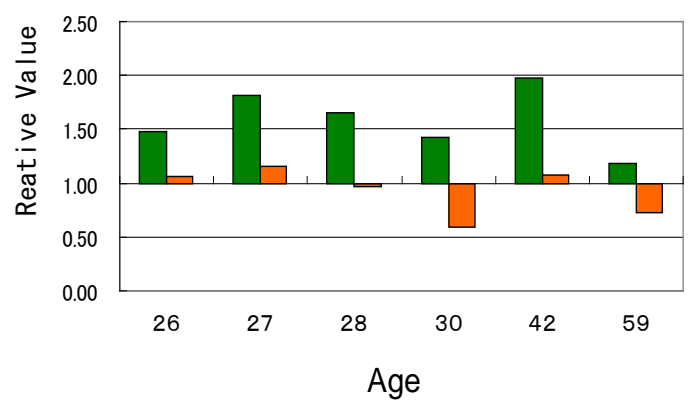

Figure 16. We tried to express the effect of peripheral total leukocyte number by individual level of change and plot in the $\mathrm{x}$-axis as in each value according to the age. The relative value (\%) of post trial was calculated with before and after and plotted in the y-axis. Leukocyte subset, granulocyte and lymphocyte were also traced in the Figures.

\section{An Importance to Regulate Neutral in Both Leucocytes Subsets in Number}

The results varied among the lymphocyte subsets before and after hot spring hydrotherapy. After hydrotherapy, the $\mathrm{CD}_{16}{ }^{+}, \mathrm{CD}^{+}$and $\mathrm{CD} 19^{+}$cells increased in the younger group. Meanwhile, the $\mathrm{CD}_{16}{ }^{+}$cells increased in the older group while the $\mathrm{CD}_{1} 9^{+}$cells decreased remarkably (Figure 14, Figure 17). This quantitative change in lymphocytes is shown in Figure 14. Except for $\mathrm{CD}^{+}$cells, the $\mathrm{CD}^{+}, \mathrm{CD}^{+}, \mathrm{CD}_{16}{ }^{+}, \mathrm{CD} 19^{+}$and $\mathrm{CD}^{2} 6^{+}$cells all showed negative variations.

There was a strong correlation between the variable ratio and the value before hydrotherapy: high values tended to decrease and low values tended to increase.

A comparison of the $\mathrm{CD} 4^{+} / \mathrm{CD} 8^{+}$cells and $\mathrm{CD} 16^{+} / \mathrm{CD} 56^{+}$cells between the two groups was shown. All the ratios of the $\mathrm{CD}_{16} / \mathrm{CD}^{+} 6^{+}$cells increased in both groups. The changes in the $\mathrm{CD} 4^{+} / \mathrm{CD}^{+}$cells, however, were different: the younger group showed a decrease while the older group showed an increase.

Hot spring hydrotherapy regulated the leukocyte count differently in $G$ - and L-type individuals. In order to clarify the effect on individuals according to the type of regulation, we divided the volunteers into two groups: the G-type group had a granulocyte count over $70 \%$ and the L-type group had a lymphocyte count over $40 \%$. Figure 11 shows that after hydrotherapy, the lymphocytes or lymphocyte subsets increased in the G-type group, accompanied by a decrease in granulocytes. However, in the L type group, the granulocytes increased notably as did the number of lymphocyte populations.

Hot spring hydrotherapy affected the functional changes in cytokine-containing cells. To test whether hot spring hydrotherapy affected the functional maturation of immunocytes, we further investigated the number of cytokine containing cells by FACS analysis. The results showed the effect of hot spring hydrotherapy on cyto- 


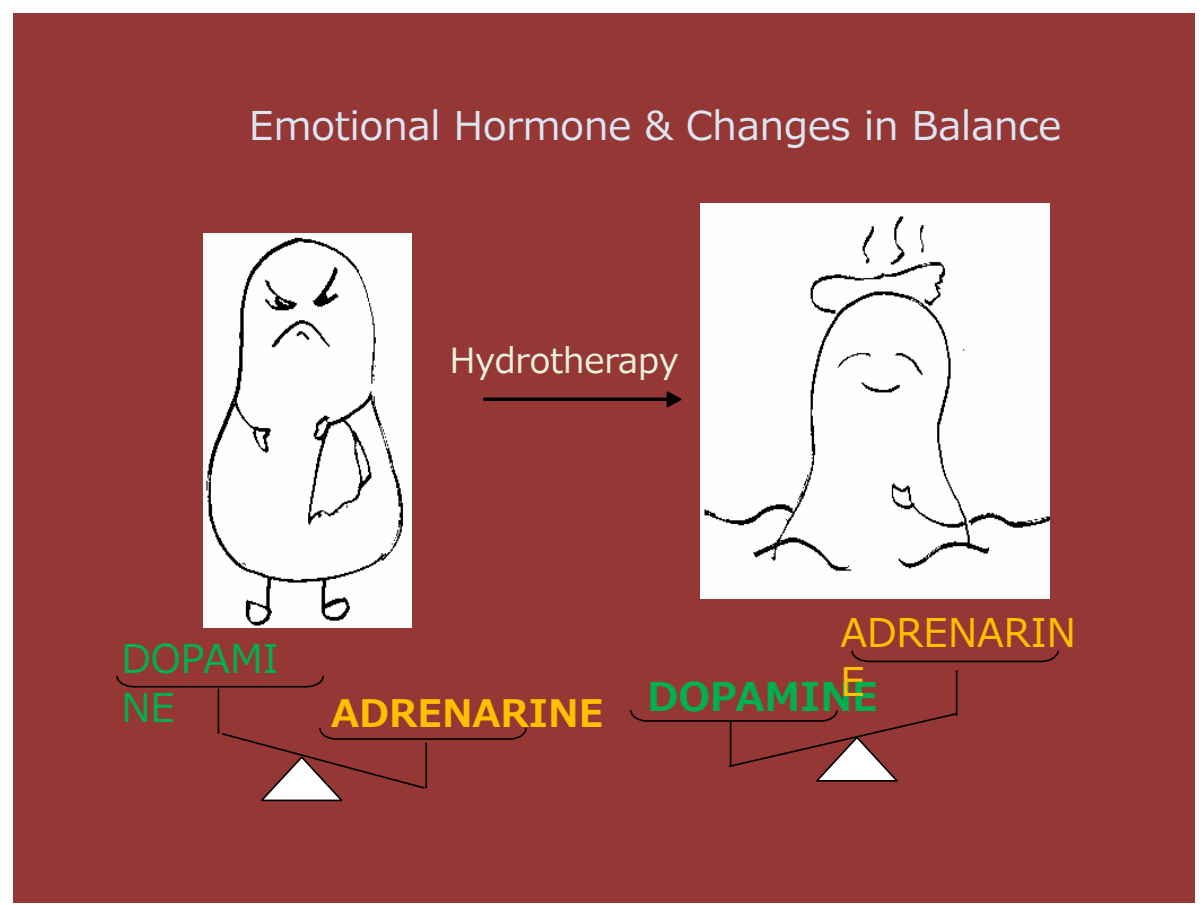

Figure 17. An illustrative presentation of hormonal regulation by hot Spring hydrotherapy. The clear slant was made from the data especially, in regulation in adrenalin, overall trend was decrease but was dose dependent manner of the value the day before. Also, dopamine was increased but also dose dependent manner as slant making.

kine production. Even though the increase in IFN- $\gamma$ containing cells had no statistical significance, the increase in IL-4 was remarkable ( $r=0.560, p<0.01)$ while the IL- $1 \beta$ containing cell counts showed a decrease. In addition, before and after hydrotherapy, the levels of IFN- $\gamma$ and IL4 or IL- $1 \beta$ also had meaningful negative changes. After hydrotherapy, there was a decrease of cytokine-producing cells in the subjects who had previously had a higher level.

The changes depended on the basic level of the white blood cells in the blood of the individuals.

Hot spring hydrotherapy changes hormonal level in blood. The peripheral blood was sampled twice from the volunteers at before and after 24 hours of the hydrotherapy. As a measurement item, thyroid hormone and 4 kinds of hormone reported to related emotion were measured. As a result of measuring the blood hormonal level around the hot spring hydrotherapy, adrenalin quantity showed the tendency in decrease in most individuals, while dopamine level showed the tendency in increase. On the other hormonal level, the fluctuation could not be observed (Figure 12). We also found a negative correlation between the variable ratio and the value before hydrotherapy in adrenalin level (Figure 12): high values tended to decrease and low values tended to increase ( $r=$ $-0.62016, p<0.001)$.

\subsection{Twenty-Four Hours Change of Total Peripheral Leukocyte Count on the Basis of Each Individual}

Each volunteer was prepared their blood before start to visit hot spring village after informed consented to the experimental purpose by written Ethics of the Committee in Kanazawa Medical University. After lodging the hotel, volunteers enjoyed Japanese type hot spring bath system, with no under wear in the bath room/pool separately prepared for ladies and gentleman. We suggested taking a bath totally 2 - 3 times within two days for the aim of trial. Beside the advance of VAS: visual analog scale in our investigation, we measured the total number of leukocyte and two major subsets, granulocyte and lymphocytes regulated before and after hot spring hydrotherapy.

We tried to express the effect of peripheral total leukocyte number by individual level of change and plot in the $\mathrm{X}$-axis as in each age. As was in Figure 3, the groups was separated in to two, up-regulated individuals and 
down-regulated one. The correlation of change was expressed as a linear function. Figure 10 was ideal change of effect with hot spring hydrotherapy, showing the best inclination. The results showed that these subsets could reflect the number and function of immuno-competent cells. For example, in an individual with a low granulocyte number, the number increased after treatment, while it decreased in another individual with a higher cell number (Figure 5 and Figure 6).

We sampled peripheral blood from the 12 volunteers before and after hot sand/hydro therapy, at the same O'clock on the day, with the respect of circadian rhythm [25] [27] of leukocyte. These subjects participated in this study after giving their informed consent. We conducted the experiment at Yuwaku Onsen Spa (Kanazawa, Ishikawa Pref., Japan) in one night of the day. The spring quality is a weak sodium chloride with sodium carbohydrate of the water temperature $41^{\circ} \mathrm{C} \pm 1{ }^{\circ} \mathrm{C}$. During the night and in the morning of the next day, they had a bath in the hot spring two or three times, for 20 - 30 minutes each time. Hot sand spa had performed and bathing for 40 mins. Time interval of blood sampling between before and after hot spring hydrotherapy was approximately 24 hours. Measurements of the total and differential leukocyte counts and 3 catecholamines levels in the peripheral blood.

We ordered on the laboratory of Ishikawa Prefecture Preventive Medicine Association about the total and differential leukocyte counts and the levels of 3 catecholamines (adrenaline, noradrenaline, and dopamine) in the peripheral blood from the subjects. The total and differential leukocyte counts were measured by the automated hematology analyzer XE-2100 (Sysmex, Inc., Kobe, Japan). The levels of catecholamines were measured by high performance liquid chromatography (HPLC) system (Tosoh Co. and Hitachi High-Technologies Co., Japan).

\subsection{Twenty-Four Hours Change of Leukocyte Counts on the Bases of Group Comparison between Pre/Post Hydrotherapy}

Despite the expression in the Section 3.1, when we tried to explain these values into the grouped value before and after the hydrotherapy, we got only one positive result from the granulocyte change that was the major population of peripheral leukocytes. The other factor did not exhibit as positive result.

The total and differential leukocyte counts in the peripheral blood of 12 subjects tended to decrease after hotspring hydro-therapy. The statistically significant result only obtained that the granulocyte counts significantly decreased $(p<0.05)$. Another minor group lymphocyte did not changed significantly by judging from simple. Hot Spring Hydro Therapy Regulate Peripheral Leukocyte Together with Emotional Hormone and Receptor Positive Lymphocytes According to Each Constitution/Condition 6.

The effect of hot spring hydrotherapy on the leukocyte count correlated with the age and original basic count of the individuals. Figure 2 shows the total numbers of leukocytes, granulocytes and lymphocytes from peripheral blood collected before and after hot spring hydrotherapy in the older and younger groups. The quantitative variation of the two groups differed. In the younger group, the total number of WBC $(p<0.05)$ and granulocytes $(p<0.01)$ clearly decreased. On the other hand, the total number of WBC and lymphocytes significantly increased $(p<0.01)$ after hydrotherapy in the older group. Furthermore, the results show that changes in the leukocyte count and subset count had a negative relationship before and after hydrotherapy. In other words, subjects who had a higher cell count level before hydrotherapy tended to show a decrease in the number of WBC 24 hrs after hydrotherapy. There was a significant correlation between age and rate of change (Figure 2, $r$ $=0.91, p<0.001)$. Since the turning point occurred at 35 years old, we separated the participants into a younger (under 35) and an older (over 36) group.

\section{Leucocytes Subsets Regulated the Number and Function}

\subsection{Twenty-Four Hours Change of Total Peripheral Leukocyte Count on the Basis of Each Individual}

Each volunteer was prepared their blood before start to visit hot spring village after informed consented to the experimental purpose by written Ethics of the Committee in Kanazawa Medical University. After lodging the hotel, volunteers enjoyed Japanese type hot spring bath system, with no under wear in the bath room/pool separately prepared for ladies and gentleman. We suggested taking a bath totally 2 - 3 times within two days for the aim of trial. Beside the advance of VAS: visual analog scale in our investigation, we measured the total number 
of leukocyte and two major subsets, granulocyte and lymphocytes regulated before and after hot spring hydrotherapy.

We tried to express the effect of peripheral total leukocyte number by individual level of change and plot in the $\mathrm{X}$-axis as in each age. As was in Figure 3, the groups was separated in to two, up-regulated individuals and down-regulated one. The correlation of change was expressed as a linear function. Figure 10 was ideal change of effect with hot spring hydrotherapy, showing the best inclination. The results showed that these subsets could reflect the number and function of immuno-competent cells. For example, in an individual with a low granulocyte number, the number increased after treatment, while it decreased in another individual with a higher cell number.

We sampled peripheral blood from the 12 volunteers before and after hot sand/hydro therapy, at the same O'clock on the day, with the respect of circadian rhythm [25] [27] of leukocyte. These subjects participated in this study after giving their informed consent. We conducted the experiment at Yuwaku Onsen Spa (Kanazawa, Ishikawa Pref., Japan) in one night of the day. The spring quality is a weak sodium chloride with sodium carbohydrate of the water temperature $41^{\circ} \mathrm{C} \pm 1{ }^{\circ} \mathrm{C}$. During the night and in the morning of the next day, they had a bath in the hot spring two or three times, for 20 - 30 minutes each time. Hot sand spa had performed and bathing for 40 mins. Time interval of blood sampling between before and after hot-spring hydrotherapy was approximately 24 hours. Measurements of the total and differential leukocyte counts and 3 catecholamines levels in the peripheral blood.

We ordered on the laboratory of Ishikawa Prefecture Preventive Medicine Association about the total and differential leukocyte counts and the levels of 3 catecholamines (adrenaline, noradrenaline, and dopamine) in the peripheral blood from the subjects. The total and differential leukocyte counts were measured by the automated hematology analyzer XE-2100 (Sysmex, Inc., Kobe, Japan). The levels of catecholamines were measured by high performance liquid chromatography (HPLC) system (Tosoh Co. and Hitachi High-Technologies Co., Japan).

\subsection{Twenty-Four Hours Change of Leukocyte Counts on the Bases of Group Comparison between Pre/Post Hydrotherapy}

Despite the expression in the Section 3.1, when we tried to explain these values into the grouped value before and after the hydrotherapy, we got only one positive result from the granulocyte change that was the major population of peripheral leukocytes. The other factor did not exhibit as positive result.

The total and differential leukocyte counts in the peripheral blood of 12 subjects tended to decrease after hotspring hydro-therapy. The statistically significant result only obtained that the granulocyte counts significantly decreased $(p<0.05)$. Another minor group lymphocyte did not changed significantly by judging from simple.

Hot Spring Hydro Therapy Regulate Peripheral Leukocyte Together with Emotional Hormone and Receptor Positive Lymphocytes According to Each Constitution/Condition.

Figure 18 shows the total numbers of leukocytes, granulocytes and lymphocytes from peripheral blood collected before and after hot spring hydrotherapy in the older and younger groups. The quantitative variation of the two groups differed. In the younger group, the total number of WBC $(p<0.05)$ and granulocytes $(p<0.01)$ clearly decreased. On the other hand, the total number of WBC and lymphocytes significantly increased $(p<$ 0.01) after hydrotherapy in the older group. Furthermore, the results show that changes in the leukocyte count and subset count had a negative relationship before and after hydrotherapy. In other words, subjects who had a higher cell count level before hydrotherapy tended to show a decrease in the number of WBC 24 hrs after hydrotherapy. There was a significant correlation between age and rate of change (Figure 2, $r=0.91, p<0.001$ ). Since the turning point occurred at 35 years old, we separated the participants into a younger (under 35 ) and an older (over 36) group.

\section{The Slant Suggest the Efficacy of Each CAM Menu}

\subsection{In Case of Hot Spring Hydrotherapy}

Each volunteer was prepared their blood before start to visit hot spring village after informed consented to the experimental purpose by written Ethics of the Committee in Kanazawa Medical University. After lodging the hotel, volunteers enjoyed Japanese type hot spring bath system, with no under wear in the bath room/pool separately prepared for ladies and gentleman. We suggested taking a bath totally 2 - 3 times within two days for the 
aim of tryal. Beside the advance of VAS: visual analog scale in our investigation, we measured the total number of leukocyte and two major subsets, granulocyte and lymphocytes regulated before and after hot spring hydrotherapy.

We tried to express the effect of peripheral total leukocyte number by individual level of change and plot in the $\mathrm{X}$-axis as in each age. As was in Figure 2, the groups was separated in to two, up-regulated individuals and down-regulated one. The correlation of change was expressed as a linear function. Figure 10 was ideal change of effect with hot spring hydrotherapy, showing ideal inclination. The results showed that these subsets could reflect the number and function of immuno-competent cells. For example, in an individual with a low granulocyte number, the number increased after treatment, while it decreased in another individual with a higher cell number.

We sampled peripheral blood from the 12 volunteers before and after hot sand/hydro therapy, at the same O'clock on the day, with the respect of circadian rhythm [21] [30] of leukocyte. These subjects participated in this study after giving their informed consent. We conducted the experiment at Yuwaku Onsen Spa (Kanazawa, Ishikawa Pref., Japan) in one night of the day. The spring quality is a weak sodium chloride with sodium carbohydrate of the water temperature $41^{\circ} \mathrm{C} \pm 1^{\circ} \mathrm{C}$. During the night and in the morning of the next day, they had a bath in the hot spring two or three times, for 20 - 30 minutes each time. Hot sand spa had performed and bathing for 40 mins. Time interval of blood sampling between before and after hot-spring hydrotherapy was approximately 24 hours. Measurements of the total and differential leukocyte counts and 3 catecholamines levels in the peripheral blood.

We ordered on the laboratory of Ishikawa Prefecture Preventive Medicine Association about the total and differential leukocyte counts and the levels of 3 catecholamines (adrenaline, noradrenaline, and dopamine) in the peripheral blood from the subjects. The total and differential leukocyte counts were measured by the automated hematology analyzer XE-2100 (Sysmex, Inc., Kobe, Japan). The levels of catecholamines were measured by high performance liquid chromatography (HPLC) system (Tosoh Co. and Hitachi High-Technologies Co., Japan).

\subsection{Emotional Hormones Also Regulated with Condition/Constitution Dependent Manner}

We had been observed the reputational effect of hydrotherapy could be evidenced within a short period. But the possibility still remain that the leukocyte change was happen to emerge for the dairy life as accidental factor,

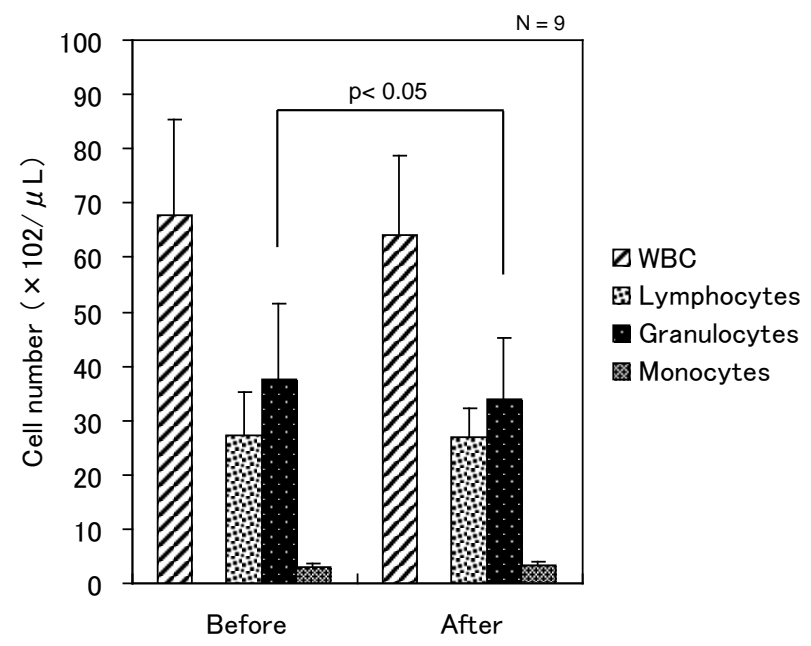

Figure 18. Twenty-four hours change of leukocyte counts on the bases of group comparison between pre/post hydrotherapy. We sampled peripheral blood from the 12 volunteers before and after hot spring hydrotherapy, at the same time on each day, in accordance with the consideration of circadian rhythm of leukocyte In this Figure, we tried to show the date simply pooled and make mean, then compared. 
such as stress and so on. In order to avoid such possibility, then we tried to show the change of the peripheral leukocyte number was the result of another network system of the inner system such as peripheral leukocyte, endocrine and brain system. We sampled peripheral blood from the 12 volunteers before and after hot sand/hydro therapy, at the same time on the next day, with the respect of circadian rhythm of leukocyte [21]. These subjects participated in this study after giving their informed consent. We conducted the experiment at Yuwaku Onsen Spa (Kanazawa, Ishikawa Pref., Japan) in one night of the day. The spring quality is a weak sodium chloride with sodium carbohydrate of the water temperature $41^{\circ} \mathrm{C} \pm 1^{\circ} \mathrm{C}$. During the night and in the morning of the next day, they had a bath in the hot spring two or three times, for 20-30 minutes each time. Time interval of blood sampling between before and after hot spring hydrotherapy was approximately 24 hours. Measurements of the total and differential leukocyte counts and 3 catecholamines levels in the peripheral blood

The adrenaline and nor-adrenaline are secreted by various stress (stimulation); the former from the adrenal medulla and the latter from both the adrenal medulla and the end of sympathetic nerve. They express these functions through the adrenergic receptors (ARs) and regulate the target organ. The adrenergic receptor has two types of $\alpha$ and $\beta$, and there are multiple subtypes $\left(\alpha_{1}, \alpha_{2}, \beta_{1}, \beta_{2}, \beta_{3}\right)(4)$. These subtypes present the vascular $\left(\alpha_{1}\right)$, the presynaptic terminal $\left(\alpha_{2}\right)$, the heart $\left(\beta_{1}\right)$, the bronchial muscle of the lung $\left(\beta_{2}\right)$, fat cells $\left(\beta_{3}\right)$, respectively. The $\alpha$-receptor stimulation causes bronchodilatation and vasodilatation, the $\beta_{1}$-receptor stimulation causes an increase in heart rate and lipolysis, and the $\beta_{2}$-receptor stimulation causes bronchoconstriction, vasodilatation, and muscle glycogen resolution. The adrenaline provides heart activation by effect on $\alpha$ - and $\beta$-receptors, and the nor-adrenaline provides blood pressure rises by strongly effect on $\alpha$-receptors. In addition to the above, it has been reported that the ARs are present on leucocyte membranes [21]-[23] and that the level of expression of $\beta$ ARs in lymphocytes was examined by radioligand ( ${ }^{125}$ iodopindolol) binding, but the details of subtype of $\beta$ - receptors have not been disclosed. The subtypes of the AR are able to be analyzed by flow cytometry (FCM) method. In this study, we described how hot spring hydrotherapy influences leukocyte, lymphocyte subpopulations expressing $\beta_{2}$-AR and the levels of catecholamine in human peripheral blood (Figure 19).

\subsection{Positive Lymphocytes Correlate with Serum Adrenaline Level, as a Result of the Regulation by Hot Spring Hydrotherapy}

The analysis of CD positive cells by FCM was measured by gating in the lymphocytes region on the scattered gram. Figure 8 shows an example analysis. Nonspecific reaction of the PE fluorescence was found in the isotype control. Therefore, the real values of the AR expressing cell counts were calculated by subtracting the control values. The $\mathrm{CD} 19^{+}$cells were observed nonspecific reaction which seems to response of the second antibody. The comparison of each lymphocyte subpopulations before and after hot spring hydrotherapy showed that the $\mathrm{CD}^{+}$cell and CD56 ${ }^{+}$cell counts tended to increase (Figure 5, Figure 6). However, the significant variation was not seen from the comparison of $\beta_{2}$-AR expressing cells before and after hot-spring hydrotherapy. The mean $\%$ of $\beta_{2}$-AR expressing cells in the lymphocyte subsets was $18 \%-19 \%$ in $\mathrm{CD}^{+}$cells, $5 \%$ in $\mathrm{CD}^{+}$cells, $57 \%-63 \%$ in CD8 ${ }^{+}$cells, and 93\% - 95\% in CD56 cells. That in CD $19^{+}$cells was approximately 100\% (data not shown), but we were not able to be confirmed because it was very likely to be the nonspecific reaction.

We examined the correlation with the rate of change in adrenaline levels and the rate of change in $\beta_{2}$-AR expressing cell counts of each subset or that in each CD-positive cell counts before and after hot spring hydrotherapy. In the CD-positive cells, the rate of change in adrenaline levels was a positive correlation with that in the levels of $\mathrm{CD}^{2} 6^{+}$cells, $\mathrm{CD}^{+}$cells, and $\mathrm{CD}^{+}$cells; in particular, a correlation with $\mathrm{CD}^{2} 6^{+}$and $\mathrm{CD}^{+}$cells was high. In $\beta_{2}$-AR expressing cells, the rate of change in adrenaline levels was a positive correlation with the rate of change in the levels of $\beta_{2}-\mathrm{AR}^{+} \mathrm{CD} 56^{+}$cells (Figure 20, Figure 21 and Figure 22). These results suggested that the variation in adrenaline levels is correlated with $\mathrm{CD} 56^{+}$cells.

\section{4. $\beta_{2}-\mathrm{AR}^{+}$Receptor Positive Lymphocytes Correlate with Adrenaline Level, as a Result of the Regulation by Hydrotherapy}

The adrenaline and noradrenaline are secreted by various stress (stimulation); the former from the adrenal medulla and the latter from both the adrenal medulla and the end of sympathetic nerve. They express these functions through the adrenergic receptors (ARs) and regulate the target organ. The adrenergic receptor has two types of $\alpha$ and $\beta$, and there are multiple subtypes $\left(\alpha_{1}, \alpha_{2}, \beta_{1}, \beta_{2}, \beta_{3}\right)$. These subtypes present the vascular $\left(\alpha_{1}\right)$, the presynaptic terminal $\left(\alpha_{2}\right)$, the heart $\left(\beta_{1}\right)$, the bronchial muscle of the lung $\left(\beta_{2}\right)$, fat cells $\left(\beta_{3}\right)$, respectively. The $\alpha$ - 


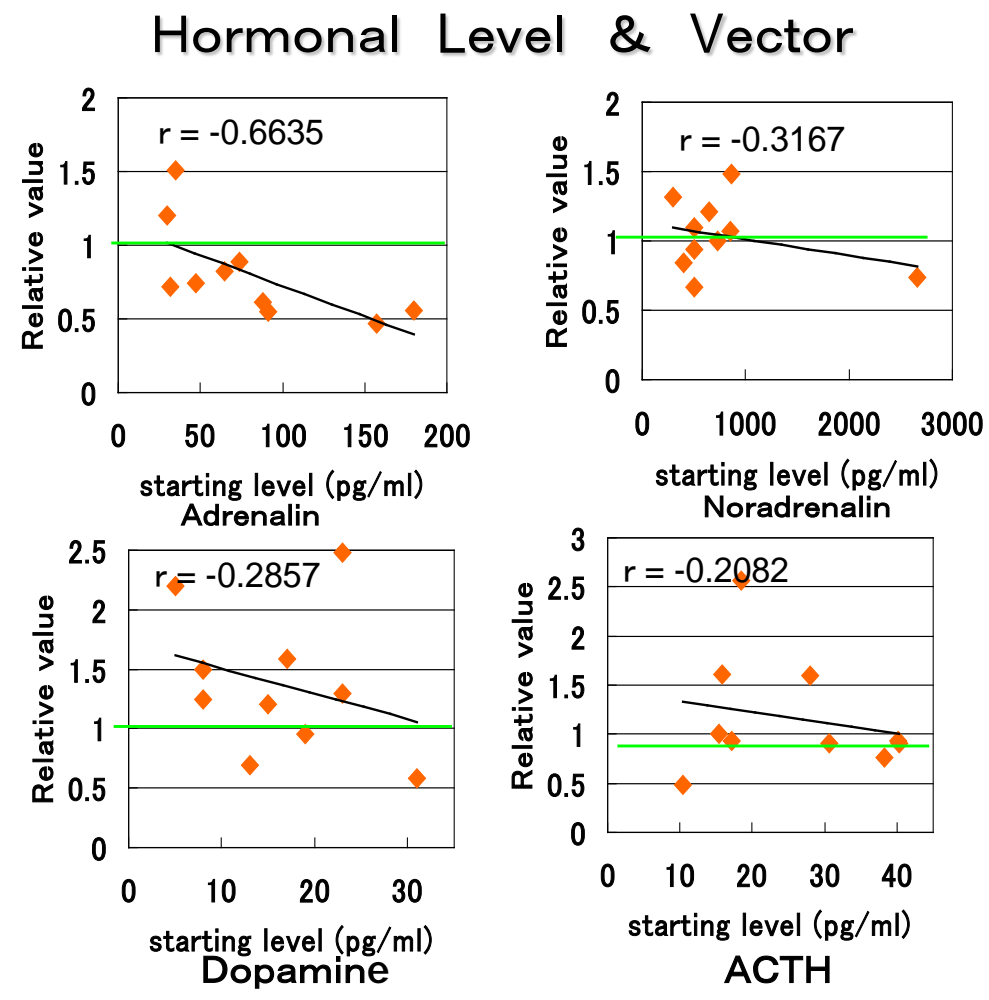

Figure 19. The constitution dependent analysis for (adrenaline and dopamine). Each spot were obtained from the calculation comparing relative value from before and after levels in the serum, catecholamines levels in the peripheral blood Relative value 1 shows no change before and after the menu. The constitution dependent analysis, the detail change and vector of each change could find from individual data, showing higher value volunteer down regulated much more than lower leveled one (Figure 20).

\section{Thyroid Hormones}

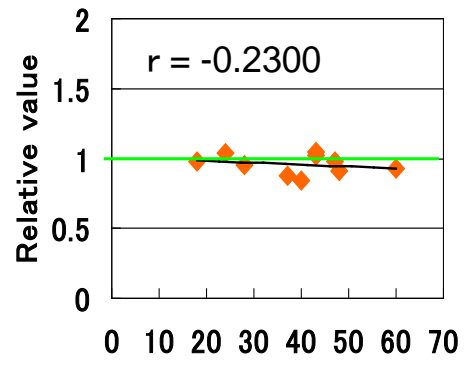

$\mathrm{T} 4(\boldsymbol{\mu g} \mathrm{g} / \mathrm{dl})$

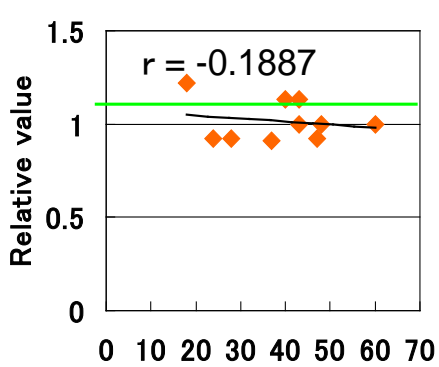

$\mathrm{T} 3(\mathbf{n g} / \mathrm{ml})$

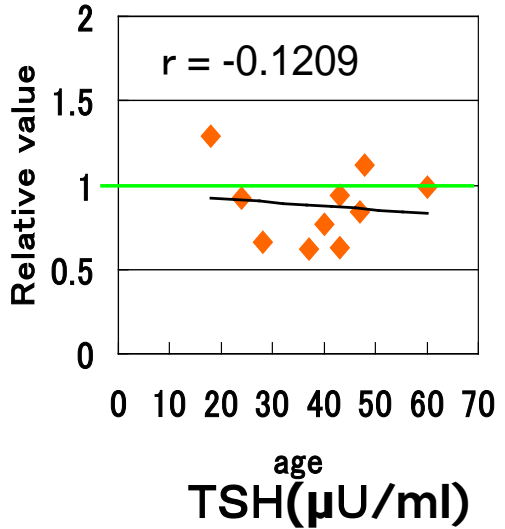

Figure 20. The constitution dependent analysis for thyroid hormones (T3, T4 and TSH). Each spot were obtained from the calculation comparing relative value from before and after levels in the serum.

receptor stimulation causes bronchodilatation and vasodilatation, the $\beta_{1}$-receptor stimulation causes an increase in heart rate and lipolysis, and the $\beta_{2}$-receptor stimulation causes bronchoconstriction, vasodilatation, and muscle glycogen resolution. The adrenaline provides heart activation by effect on $\alpha$ - and $\beta$-receptors, and the noradrenaline provides blood pressure rises by strongly effect on $\alpha$-receptors. In addition to the above, it has been 


\section{Before}
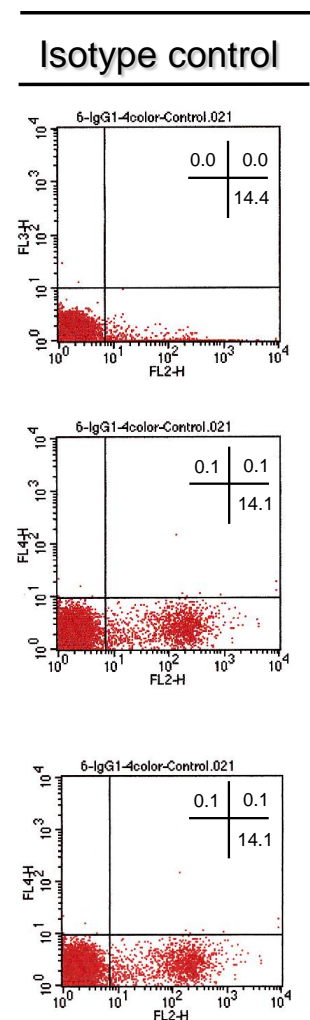

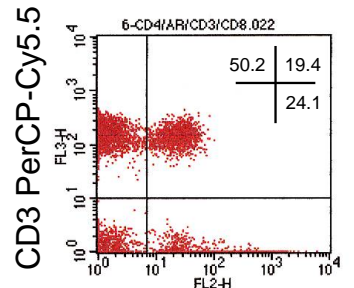

$\beta_{2}$-AR PE

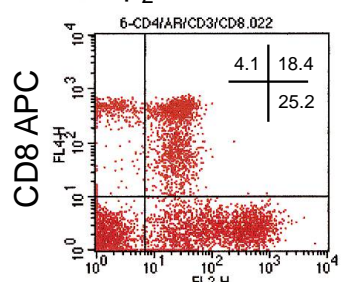

$\beta_{2}$-AR PE

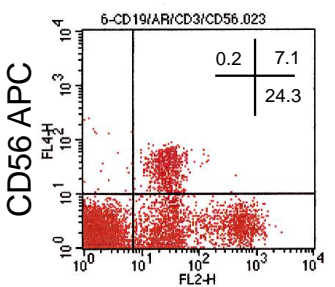

$\beta_{2}$-AR PE
After
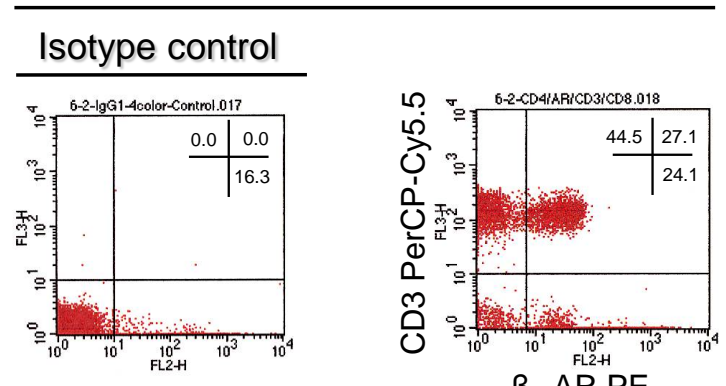

$\beta_{2}$-AR PE
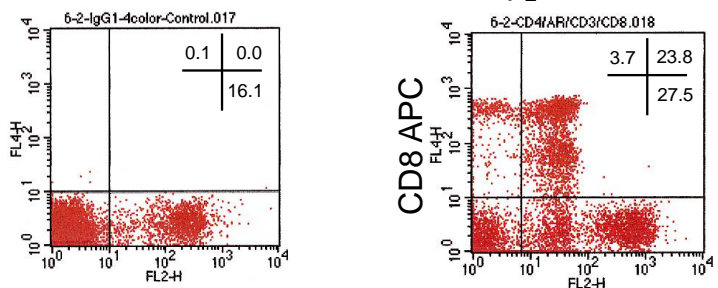

$\beta_{2}$-AR PE
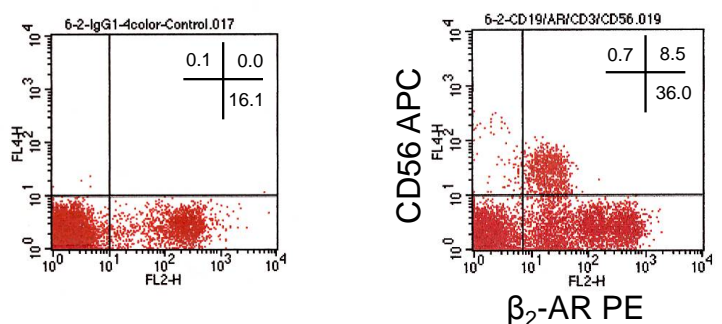

Figure 21. FACS analyses of $\beta_{2}-\mathrm{AR}^{+}$cells in lymphocyte subpopulations before and after hot spring hydrotherapy. The analysis of $\beta_{2}$-AR expressing cells and CD positive cells by FCM was measured by gating in the lymphocytes region on the scattered gram.

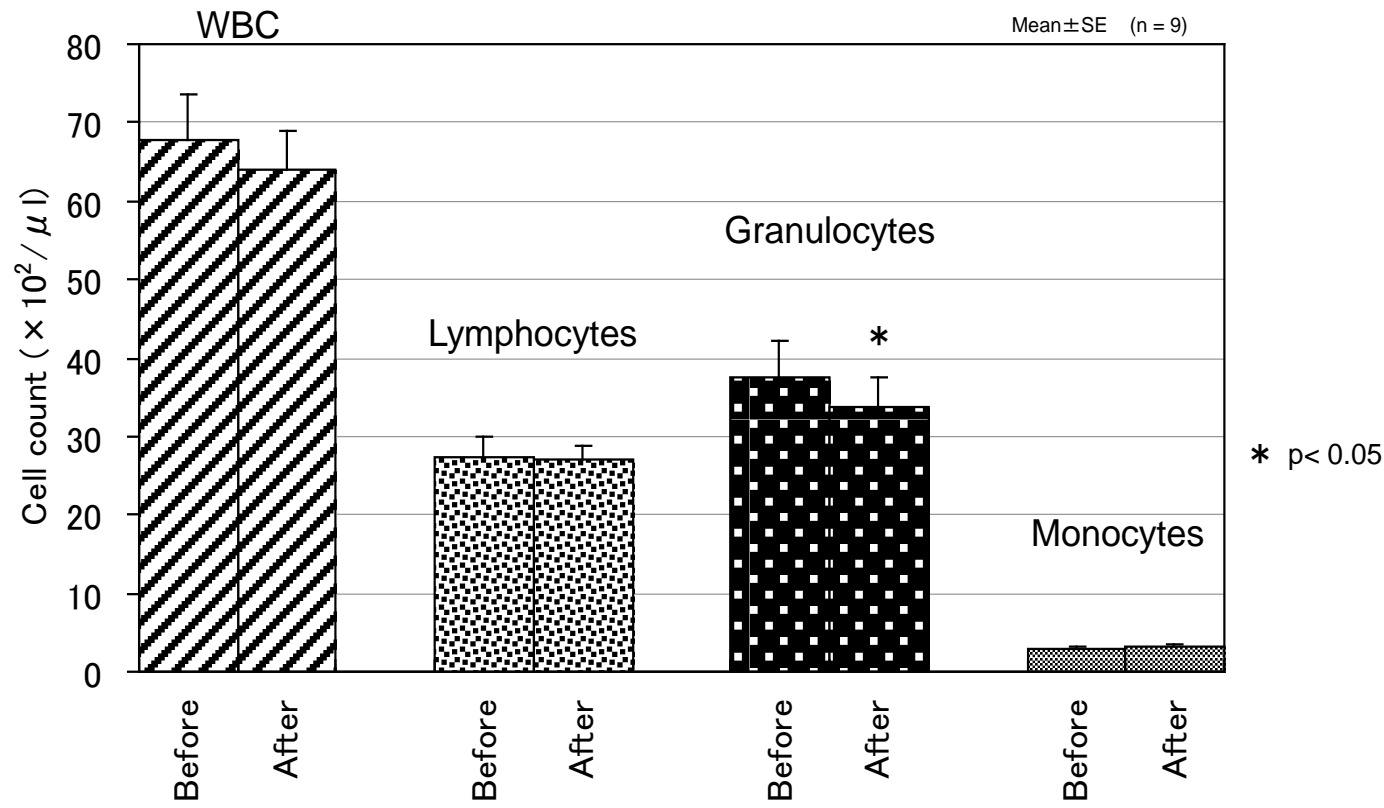

Figure 22. Non-constitutional Comparison of WBC and Subsets Number by Hydrotherapy. 

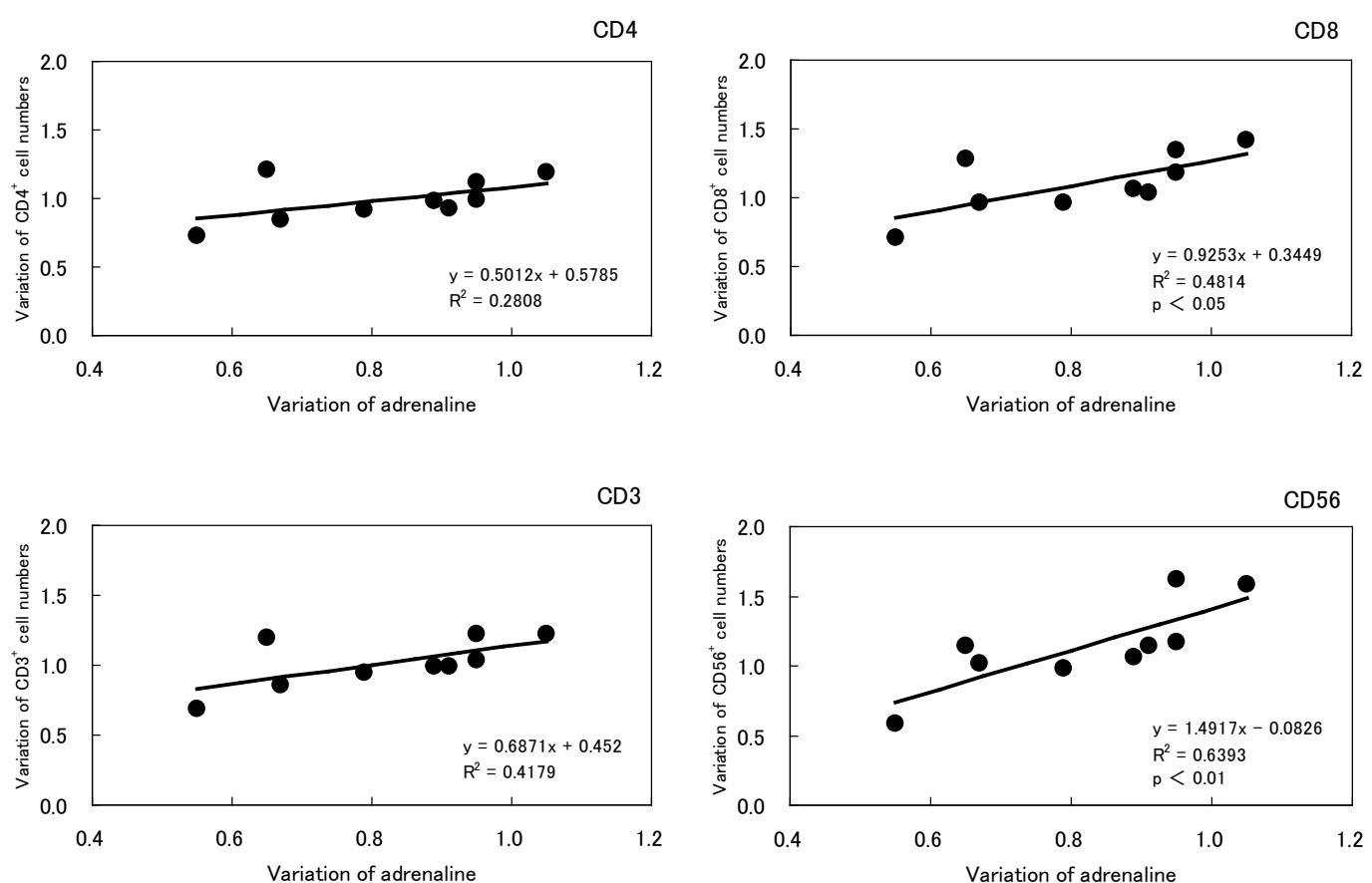

Figure 23. Relationship between the rate of change in adrenaline levels and the rate of change in the levels of CD-positive cells before and after hydrotherapy.
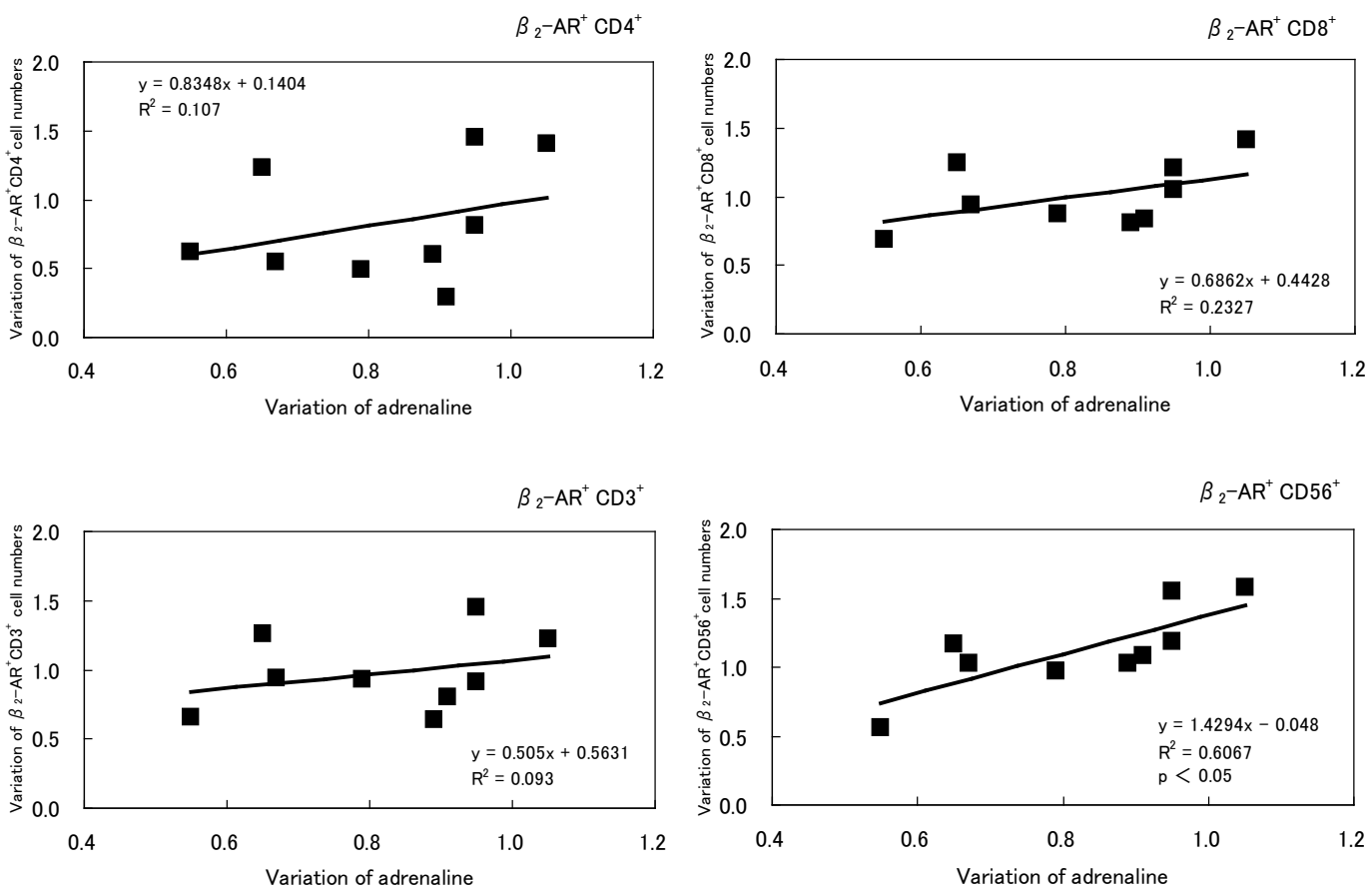

Figure 24. Variations in the number of $\beta_{2}-\mathrm{AR}^{+}$cells in lymphocyte subpopulations. And adrenaline levels in the peripheral The analysis of $\beta_{2}$-AR receptor expressing cells and CD positive cells by FCM was measured by gating in the lymphocytes region on the scattered gram. s an example analysis (Figure 23, Figure 24).

reported that the ARs are present on leucocyte membranes and that the level of expression of $\beta$-ARs in lymphocytes was examined by radioligand $\left({ }^{125}\right.$ I-iodopindolol) binding, but the details of subtype of $\beta$-receptors have not been disclosed. The subtypes of the AR are able to be analyzed by flow cytometry (FCM) method. In this study, 
we described how hot spring hydrotherapy influences leukocyte, lymphocyte subpopulations expressing $\beta_{2}$-AR and the levels of catecholamine in human peripheral blood (Figure 25).

The analysis of $\beta_{2}$-AR expressing cells and CD positive cells by FCM was measured by gating in the lymphocytes region on the scattered gram. Figure showed an example analysis. Nonspecific reaction of the PE fluorescence was found in the isotype control. Therefore, the real values of the AR expressing cell counts were calculated by subtracting the control values. The $\mathrm{CD} 19^{+}$cells were observed nonspecific reaction which seems to response of the second antibody. The comparison of each lymphocyte subpopulations before and after hot spring hydrotherapy showed that the $\mathrm{CD}^{+}$cell and $\mathrm{CD}_{5}{ }^{+}$cell counts tended to increase. However, the significant variation was not seen from the comparison of $\beta_{2}$-AR expressing cells before and after hot-spring hydrotherapy. The mean $\%$ of $\beta_{2}$-AR expressing cells in the lymphocyte subsets was $18 \%-19 \%$ in CD $^{+}$cells, $5 \%$ in CD4 $4^{+}$ cells, $57 \%-63 \%$ in $\mathrm{CD}^{+}$cells, and $93-95 \%$ in CD56 ${ }^{+}$cells. That in $\mathrm{CD}^{+} 9^{+}$cells was approximately $100 \%$ (data not shown), but we were not able to be confirmed because it was very likely to be the nonspecific reaction.

We examined the correlation with the rate of change in adrenaline levels and the rate of change in $\beta_{2}$-AR expressing cell counts of each subset or that in each CD-positive cell counts before and after hot spring hydrotherapy. In the CD-positive cells, the rate of change in adrenaline levels was a positive correlation with that in the levels of $\mathrm{CD}^{+} 6^{+}$cells, $\mathrm{CD}^{+}$cells, and $\mathrm{CD}^{+}$cells; in particular, a correlation with $\mathrm{CD}^{+} 6^{+}$and $\mathrm{CD}^{+}$cells was high (Figure 20). In $\beta_{2}$-AR expressing cells, the rate of change in adrenaline levels was a positive correlation with the rate of change in the levels of $\beta_{2}-\mathrm{AR}^{+} \mathrm{CD}_{5}{ }^{+}$cells. These results suggested that the variation in adrenaline levels is correlated with $\mathrm{CD}^{+} 6^{+}$cells.

\section{The Results in Light Exercise/Walking}

\section{Emotional Hormones Also Regulated with Condition/Constitution Dependent Manner}

We had been observed the reputational effect of hydrotherapy could be evidenced within a short period. But the possibility still remain that the leukocyte change was happen to emerge for the dairy life as accidental factor, such as stress and so on. In order to avoid such possibility, then we tried to show the change of the peripheral leukocyte number was the result of another network system of the inner system such as peripheral leukocyte, endocrine and brain system. We sampled peripheral blood from the 12 volunteers before and after hot sand/hydro therapy, at the same time on the next day, with the respect of circadian rhythm of leukocyte [25]-[27]. These subjects participated in this study after giving their in- formed consent. We conducted the experiment at Yuwaku Onsen Spa (Kanazawa, Ishikawa Pref., Japan) in one night of the day. The spring quality is a weak sodium chloride with sodium carbohydrate of the water temperature $41^{\circ} \mathrm{C} \pm 1^{\circ} \mathrm{C}$. During the night and in

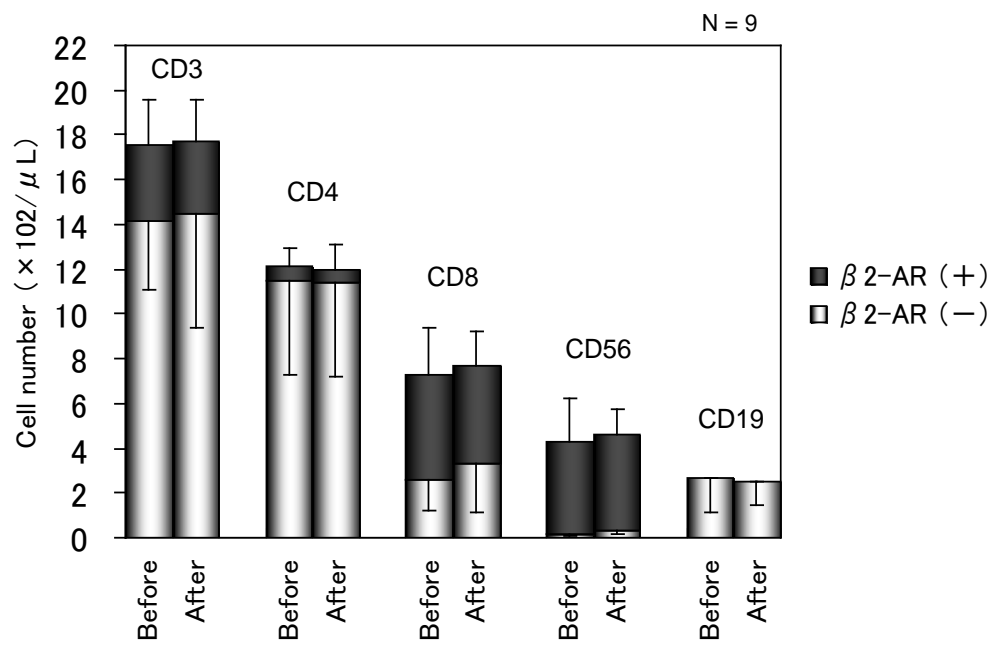

Figure 25. Individual comparison of adrenalin levels and CD positive cells in the peripheral blood Time interval of blood sampling between before and after hot spring hydrotherapy was approximately 24 hours. Measurements of the total and differential leukocyte counts and 3 adrenergic receptors (ARs) levels in the peripheral blood. 
the morning of the next day, they had a bath in the hot spring two or three times, for 20 - 30 minutes each time. Time interval of blood sampling between before and after hot spring hydrotherapy was approximately 24 hours. Measurements of the total and differential leukocyte counts and 3 catecholamines levels in the peripheral blood.

The adrenaline and noradrenaline are secreted by various stress (stimulation); the former from the adrenal medulla and the latter from both the adrenal medulla and the end of sympathetic nerve. They express these functions through the adrenergic receptors (ARs) and regulate the target organ. The adrenergic receptor has two types of $\alpha$ and $\beta$, and there are multiple subtypes $\left(\alpha_{1}, \alpha_{2}, \beta_{1}, \beta_{2}, \beta_{3}\right)$ [21]. These subtypes present the vascular $\left(\alpha_{1}\right)$, the pre-synaptic terminal $\left(\alpha_{2}\right)$, the heart $\left(\beta_{1}\right)$, the bronchial muscle of the lung $\left(\beta_{2}\right)$, fat cells $(\beta 3)$, respectively. The $\alpha$-receptor stimulation causes bronchodilatation and vasodilatation, the $\beta 1$-receptor stimulation causes an increase in heart rate and lipolysis, and the $\beta_{2}$-receptor stimulation causes bronchoconstriction, vasodilatation, and muscle glycogen resolution. The adrenaline provides heart activation by effect on $\alpha$ - and $\beta$-receptors, and the noradrenaline provides blood pressure rises by strongly effect on $\alpha$-receptors. In addition to the above, it has been reported that the ARs are present on leucocyte membranes [22]-[24] and that the level of expression of $\beta$-ARs in lymphocytes was examined by radioligand (125I-iodopindolol) binding, but the details of subtype of $\beta$-receptors have not been disclosed. The subtypes of the AR are able to be analyzed.

The adrenaline and noradrenaline are secreted by various stress (stimulation); the former from the adrenal medulla and the latter from both the adrenal medulla and the end of sympathetic nerve. They express these functions through the adrenergic receptors (ARs) and regulate the target organ. The adrenergic receptor has two types of $\alpha$ and $\beta$, and there are multiple subtypes $\left(\alpha_{1}, \alpha_{2}, \beta_{1}, \beta_{2}, \beta_{3}\right)$ [21]. These subtypes present the vascular $\left(\alpha_{1}\right)$, the pre- synaptic terminal $\left(\alpha_{2}\right)$, the heart $\left(\beta_{1}\right)$, the bronchial muscle of the lung $\left(\beta_{2}\right)$, fat cells $\left(\beta_{3}\right)$, respectively. The $\alpha$-receptor stimulation causes bronchodilatation and vasodilatation, the $\beta 1$-receptor stimulation causes an increase in heart rate and lipolysis, and the $\beta_{2}$-receptor stimulation causes bronchoconstriction, vasodilatation, and muscle glycogen resolution. The adrenaline provides heart activation by effect on $\alpha$ - and $\beta$-receptors, and the noradrenaline provides blood pressure rises by strongly effect on $\alpha$-receptors. In addition to the above, it has been reported that the ARs are present on leucocyte membranes [22]-[24] and that the level of expression of $\beta$-ARs in lymphocytes was examined by radioligand (125I-iodopindolol) binding, but the details of subtype of $\beta$-receptors have not been disclosed. The subtypes of the AR are able to be analyzed by flow cytometry (FCM) method. In this study, we described how hot spring hydrotherapy influences leukocyte, lymphocyte subpopulations expressing $\beta_{2}$-AR.

\section{In Case of Light Exercise/Walking, for the Best Menu to Each Freak}

We selected 14 healthy volunteers (mean age, $41 \pm 15.2$ years, ranging 19 - 60 years old in both sexualities) and informed consented according to the Ethics Committee of Kanazawa Medical University. The contents of sexuality were $56.25 \%$ of the lady and $43.75 \%$ for gentleman. They were the students of Medical University and the stuff for the school of medicine. None of them was a specialist for athletic field. The group was set up into two groups according to the intense of walking, 4 mets and 8 mets.

\subsection{Walking Exercise by 4 Mets}

These subjects participated in this study after giving their informed consent. We conducted the walking exercise at the country side of Japan. The course of exercise located at 40 meter in high and $1015 \mathrm{mb}$ of atmospheric pressure. We sampled peripheral blood from the 14 volunteers before and after exercise, at the same time on each day, in consideration of circadian rhythm [21] [22] of leukocyte. The exercise start at ten o'clock for about one hour, corresponding 4 metabolic rates; mets [23] [24]. Time interval of blood sampling between before and after exercise was approximately 24 hours. Measurements of the total leukocyte were assessed for differential leukocyte counts and granlocyte and lymphocyte ratio in the peripheral blood.

We ordered to the laboratory of Ishikawa Prefecture Preventive Medicine Association for precise counts for the total and differential leukocyte counts in the peripheral blood from the subjects. The total and differential leukocyte counts were measured by the automated hematology analyzer XE-2100 (Sysmex, Inc., Kobe, Japan).

\subsection{Walking Exercise by 8 Mets}

After two weeks of cooling down for all volunteers, we set up again for the same exercise except for the intense of walking was $8 \mathrm{~km} /$ hour for the same kilo-meter of the same course (8 mets). We prepared peripheral blood 
from the same 14 volunteers before and after the exercise, at the same time on each day, in consideration of circadian rhythm [21] [30] of leukocyte. We conducted the walking exercise at the same course in country side of Japan (Figure 26).

\subsection{Assessment of Lymphocyte Subsets by FCM}

For the purpose of estimating CD positive cell, the whole blood obtained from the subjects by blood collection tube containing an anticoagulant EDTA-2K. $100 \mu \mathrm{l}$ of whole blood were added the antibody. After washing with PBS, the suspensions were mixed phycoerythrin (PE)-conjugated streptavidin (Beckman Coulter Inc. France) and fluorescence monoclonal antibody: peridinin chlorophyll protein-cyanin 5.5 (PerCP-Cy5.5)-conjugated CD2, flu conjugated CD19, FITC-conjugated CD57 (each Becton Dickinson Co. USA), allophycocyanin (APC)-conjugated CD8, and APC-conjugated CD57 (each Beckman Coulter). The negative controls were added PE-conjugated streptavidin and the isotype control antibodies to the CD antibodies. After incubation for 30 minutes at $4^{\circ} \mathrm{C}$, these samples were hemolyzed using a 10-times dilution FACS Lysing Solution (Becton Dickinson). After washing with PBS, the cell suspensions were fixed using a 10-times dilution CellFIX (Becton Dickinson) and analyzed by flow cytometer FACS Caliber (Becton Dickinson) [25] [27].

\subsection{Statistical Analysis}

The statistical comparisons between two groups (before and after walking) for the test of significant difference were performed using paired t-test and wilcoxon signed-ranks test. Further, the test of the correlation were performed a spearman's correlation coefficient by rank test. Data are expressed as means \pm standard error of mean (SE). A $p$ value $<0.05$ was considered to be statistically significant. The Kendall tau rank correlation and the two-sided $p$-value $\left(\mathrm{H}_{0}\right.$ : tau $\left.=0\right)$. The ordinary scatterplot and the scatterplot between ranks of $\mathrm{X} \& \mathrm{Y}$ are also shown [28].

\subsection{Twenty-Four Hours Change of Leukocyte Counts after 4 Mets of Walking}

Each volunteer was prepared their blood before start for exercise and after informed consented to the experimental purpose by written Ethics of the Committee in Kanazawa Medical University. After warming up for 5 minutes by Radio exercise by NHK. We suggested taking a walk for one hour for $4 \mathrm{~km}$ of the curse (4 mets). We tried to exhibit the effect of peripheral total leukocyte number by individual level of change and plot in the $\mathrm{X}$-axis as in each value before the exercise. As was in Figure, the relative value (\%) was calculated before and after the exercise and plotted in the Figures of the X-axis according to the value before exercise. As a result of the exhibition, there found three groups, separated, up-regulated individuals and down-regulated one and was no change. The correlation of change was expressed as a linear function and significant reverse correlation, -0.6893 indicating ideal value of correlative index -0.5 (Figure 27). The data obtained from 4mets exercise was brought ideal regulation. However, 8 mets could not bring such an effect significantly. About the leukocyte suborescein isothiocyanate (FITC)-conjugated CD4, FITC-conjugated CD8, FITC-conjugated CD16, FITC-

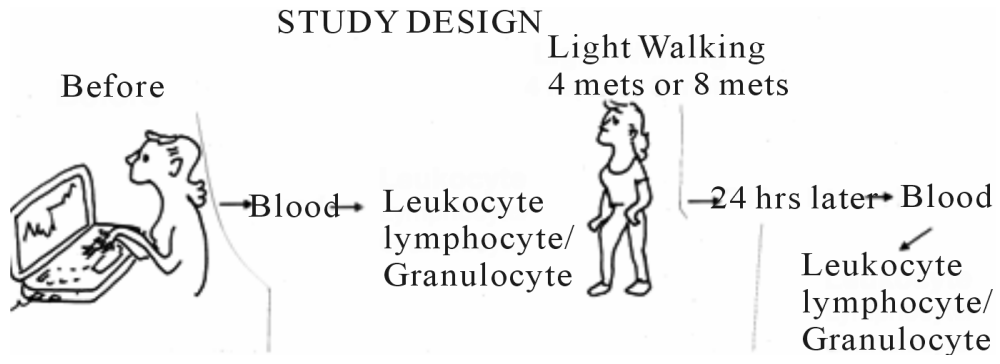

Figure 26. Experimental design of this report. We sampled peripheral blood from the 16 volunteers before and after walking exercise, at the same time on each day, in accordance with the consideration of circadian rhythm of leukocyte. We sampled peripheral blood from the 14 volunteers before and after exercise, at the same time on each day, in consideration of circadian rhythm of leukocyte. These subjects participated in this study after giving them informed consent. 
set, the ideal regulation of lymphocyte and monocyte were found by 4 mets, but not in granulocyte (Figure 27).

\subsection{Twenty-Four Hours Change of Leukocyte Counts after 8 Mets of Walking}

After 2-weeks of cooling down, we set up the same mode of trial at the same course and by the same volunteers. None of volunteer was dropped out with any serious problem. In this time, we set up 8 mets impacts of exercise, asking to walk out by half an hour for the same course of 4 kilometers (8 mets). Preparing 5-min. warming up, we tried to express the effect of peripheral total leukocyte number by individual level of change and plot in the $\mathrm{x}$-axis as in each value before the exercise as is in 3.1. (Figure 27). As shown in lower panel in Figure 28, the correlation was exhibited on a linear function but good correlation was retreated from the results by the case by 8 mets. The slant value was -0.3617 . The data obtained from 8mets exercise was lesser regulatory effect than that compared by 4 mets of exercise. About the leukocyte subset, the regulation by lymphocytes were more remarkable. However, only of the monocyte exhibited significant change by both impact 4 and 8 mets.

\subsection{Lymphocyte Subsets Regulation by 4 Mets}

We had been observed the reputational effect of exercise could be evidenced within a short period. But the possibility still remain that the leukocyte change was happen to emerge for the dairy life as accidental factor, such as stress and so on. In order to avoid such possibility, then we tried to show the change of the peripheral leukocyte number was the result of another network system of the inner system such as peripheral leukocyte, endocrine and brain system. We tried to access the effect CD positive lymphocyte. They were CD2, CD CD4, CD8, CD16, CD19, CD57 within those CD positive cells, CD2, CD4, CD8, CD19 cells were regulated significantly as in number of each CD marker positive cells by 4 mets of excercise.

\subsection{Twenty-Four Hours Change of Lymphocyte Subsets by 8 Mets}

After 2-weeks of cooling down, we set up the same mode of trial at the same course and by the same volunteers. None of volunteer was dropped out with any serious problem. In this time, we set up 8 mets impacts of exercise, asking to walk out by half an hour for the same course of 4 kilometers (8 mets). Preparing 5-min. warming up, we tried to express the effect on lymphocyte subsets as CD positive number. They were CD2, CD 4, CD 8, CD 16, CD 19, CD57. As shown in the Figure 29, the regulatory effect was overwealed 27 in the all the subset in 8 mets of exercise.

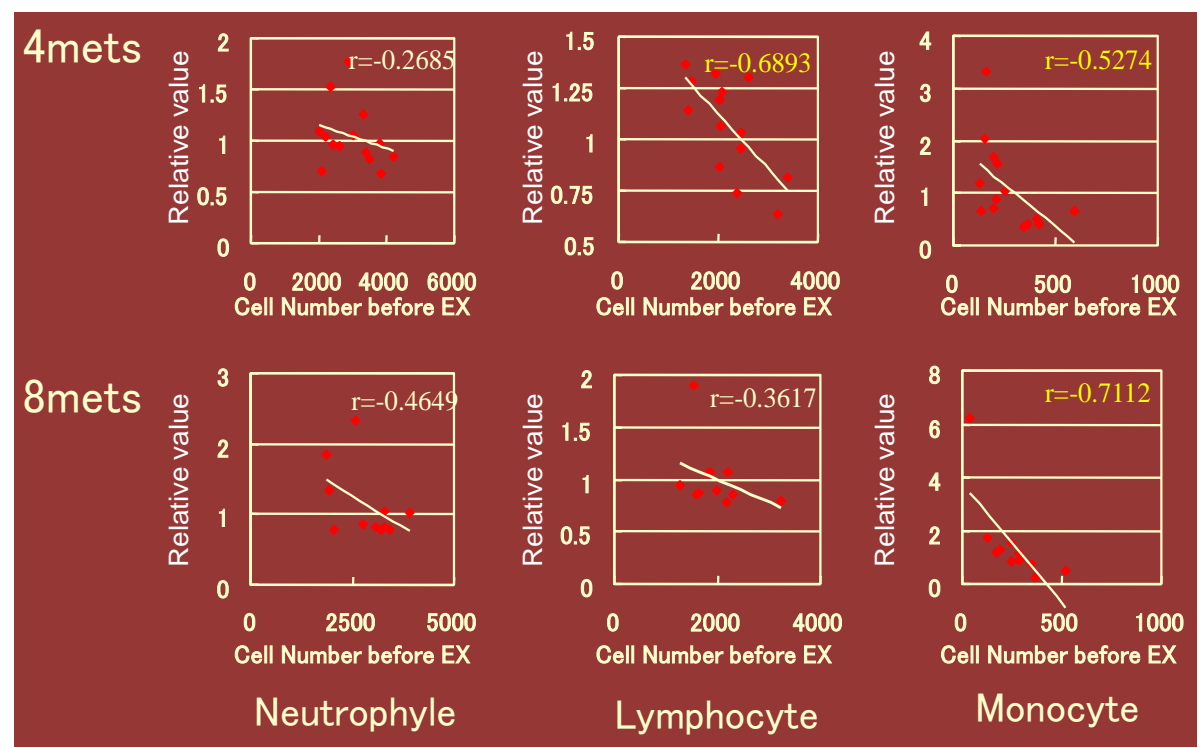

Figure 27. We tried to express the effect of peripheral total leukocyte number by individual level of change and plot in the $\mathrm{x}$-axis as in each value before the exercise. The relative value (\%) of post walking was calculated with before and after and plotted in the X-avis. The statistically significant value of slope were Indicated in yellow. 


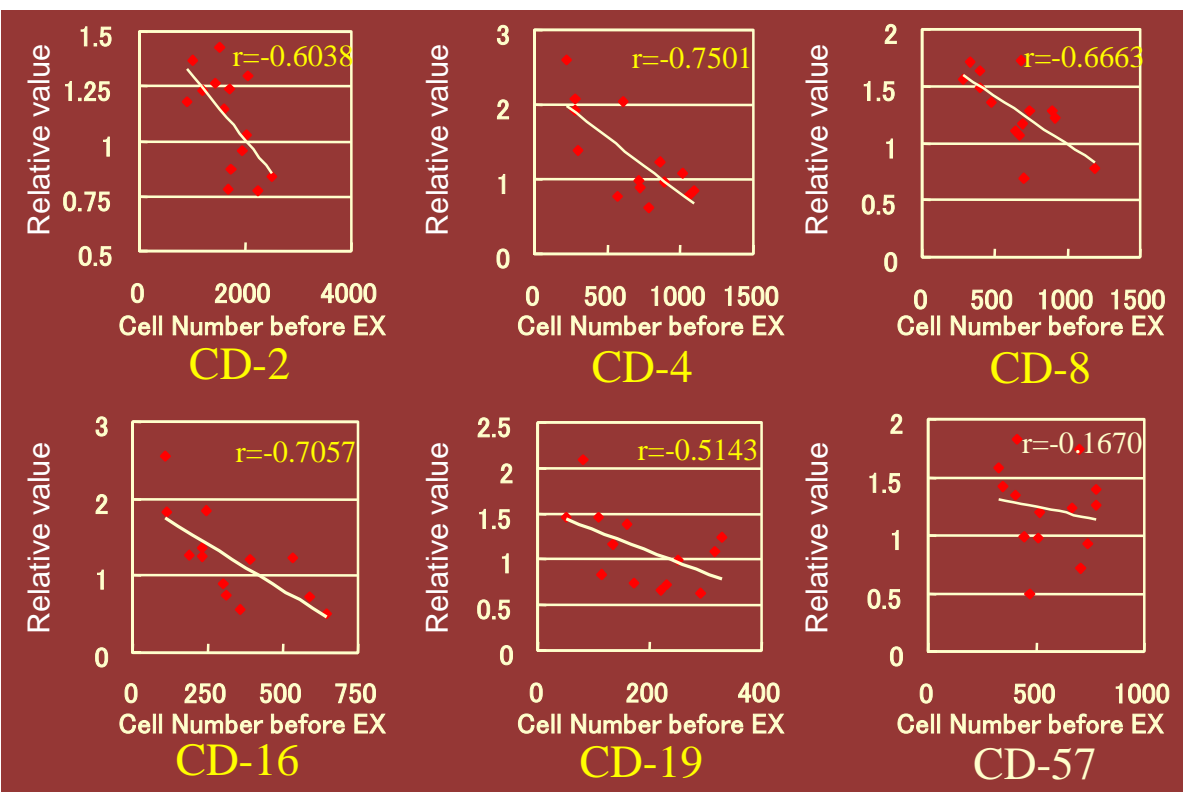

Figure 28. For the purpose of estimating CD positive cell, the whole blood obtained from the subjects by blood collection tube containing anticoagulant EDTA-2K. $100 \mu \mathrm{l}$ of whole blood were added the antibody. After washing with PBS, the suspensions were mixed phycoerythrin (PE)-conjugated streptavidin: peridinin chlorophyll protein-cyanin 5.5 (PerCP-Cy5.5)-conjugated CD2, CD4, CD8, CD16, CD19, CD57. We tried to express the effect of peripheral total leukocyte number by individual level of change and plot in the $\mathrm{x}$-axis as in each value before the exercise. The relative value was calculated before and after the exercise and plotted in the figure of the $\mathrm{X}$-axis for the value before exercise. The statistically significant value of slope were indicated in yellow.

\subsection{Simple Sum up \& Make Mean, Get No Meaning after 24 Hours Change of Lymphocyte Subsets by Both 4 \& 8 Mets}

As shown in Figure 27 and Figure 28, we can compared and got the result that of 4mets of exercise was more effective to regulate lymphocyte and lymphocyte subset by individual change by plotting the linear function. We tried to exhibit the same data with grouped and tried to make mean. From the results, there was no significant change.

\subsection{The Tailored Assessment Was Provided by Linear Slant}

Our results showed that within 24 hours after light exercise, the white blood cells in peripheral blood had regulated significantly, not only in leukocyte subset but also lymphocyte subset. The results showed that these subsets could reflect the number and function of immuno-competent cells [13]-[19]. For example, in an individual with a low granulocyte number, the number increased after treatment, while it decreased in another individual with a higher cell number. Our results led us to believe that leukocyte subsets could be an interesting indicator for the evaluation of alternative therapies. Many systems are in place to evaluate Western therapies that aim at healing the symptoms of an illness. We hope that our work will attract more attention to the mechanisms of which each CAM menu regulates the human immune system. Abo reported that according to the lymphocyte subset content, lymphocyte rich type showed over $40 \%$ on the other hand granulocyte rich type show over $60 \%$ of granulocyte. Each type exhibited different character even in the same age, sexuality and different age. Within the same age and the sexuality, even in gentleman can sorts out as G-rich type (granulocyte 60\%), and L-rich type (lymphocyte $40 \%$ ). On the other hand, as a stand point of sexuality difference, the lady belongs to L-rich type but the gentleman belongs G-rich type. According to the age-related change, G-rich type of young man change to L-rich type according to getting older [21] [29].

We have been trying to regulate the immune responsiveness through much mature by fragile daily condition from circumstance stress and so on. The main menu were, acupuncture, hot-spring hydrotherapy, light exercise 
etc. In this article, we would like to show the regulatory mechanism of the light exercise walking as a tailored scale. The circumstance, except for cases of contraindication, has been medically useful approved to be effective in many stress-related disorders and the improvement of dysfunction of the biological rhythm disturbance as well as chronic disease. The mechanism of effects has been reported in many studies, but many things are still unclear.

\subsection{The Grouped Comparison by Conventional Assessment}

In order to assess correctly to the changes after the menu, it is important to conscious of circadian rhythm. Abo also reported that it was possible to sort the constitution, granulocyte-rich individual and lymphocyte-rich one with the peripheral leukocyte [22] [31]-[33].

Each population of subset is depends on a circadian rhythm. Within a same individual, granulocyte increase in the daytime, on the other hand, lymphocyte increased in the night time in a cycle 24 hrs. So we have to compare the effect of each menu for the peripheral leukocyte on the same time before and after the menu.

With our report, simple comparison of grouped value did not exhibit the valuable change in each individual. So this style of presentation was suggestive to the patient for immunodeficy such as in DM [34]-[56].

It was reported that the leukocyte subset, granulocyte and lymphocyte regulated by various factors. One major point is that they are regulated by autonomous nervous system, resulting in circadian rhythm [23] [24]. Therefore, in order to access the effect within a short time, it is necessary to consider this factor to adjust the time to collect the sample. For example, efficacy and impact of walking exercise has been widely recognized. However, the majority of walkers did not have a scientific background to know the best exercise menu for the one' QOL; quality of life. The purpose of this study was to demonstrate the best menu of walking that regulates the peripheral white blood cells in number and function as a marker of QOL expression [21] [57].

However, almost all the judgments of efficacy are VAS (visual analog scale). Moreover, simple processing by grouped value and make mean fadeout the precise regulation according to each constitution. Therefore, we hope our tailored scale can suggest to assess every CAM therapies in the world for competition.

\subsection{Twenty-Four Hours Change of Leukocyte Counts after Heating by Hot Air from Overhead}

None of volunteer was dropped out with any serious problem. In trial, we set up room temperature also 18C at

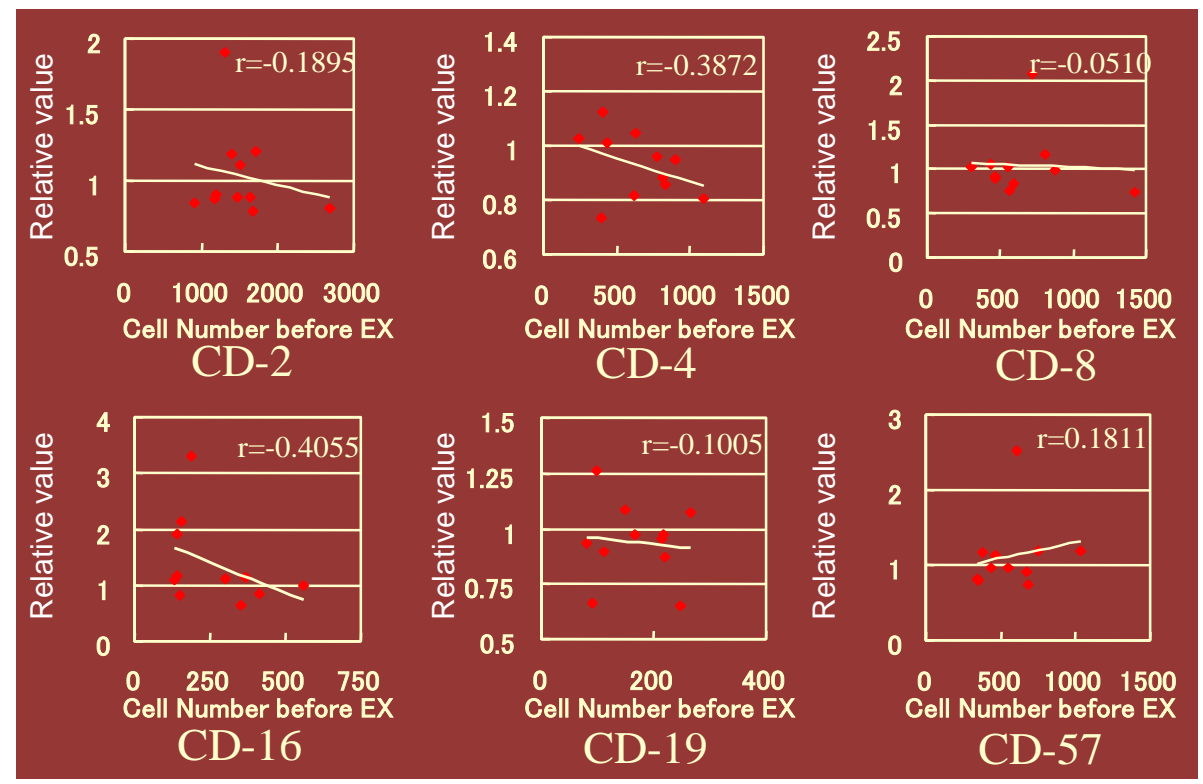

Figure 29. For the purpose of estimating CD positive cell, the whole blood obtained from the subjects by blood collection tube containing an anticoagulant EDTA-2K. The contents were the same in Figure 4 except that the data were from the volunteers from 8 mets of walking. The statistically significant value of slope were indicated in yellow. 
the $150 \mathrm{~cm}$ in high from floor, we tried to express the effect of peripheral total leukocyte number by individual level of change and plot in the X-axis as in each value before the exercise as is in 3.1. As shown in lower panel in Figure 30, the correlation was exhibited on a linear function but good correlation was retreated from the results. About the leukocyte subset, the regulation by lymphocytes were more remarkable. However, only the
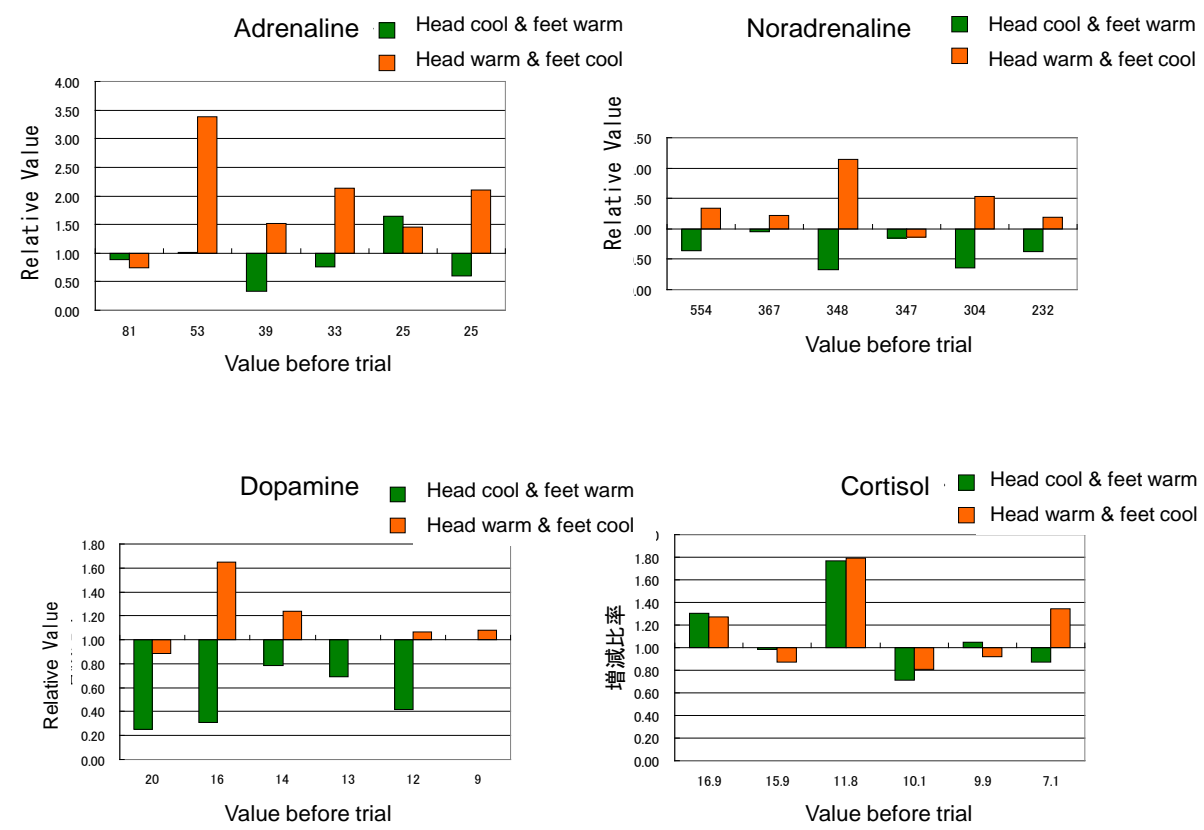

Figure 30. We tried to express the effect of peripheral total leukocyte number by individual level. The results were plotted in the $\mathrm{X}$-axis as in each value before the trial. The relative value (\%) of post trial was calculated with before and after and plotted in the Y-axis. Total leukocytes, Leukocyte subset, granulocyte and lymphocyte were also traced in the Figures.
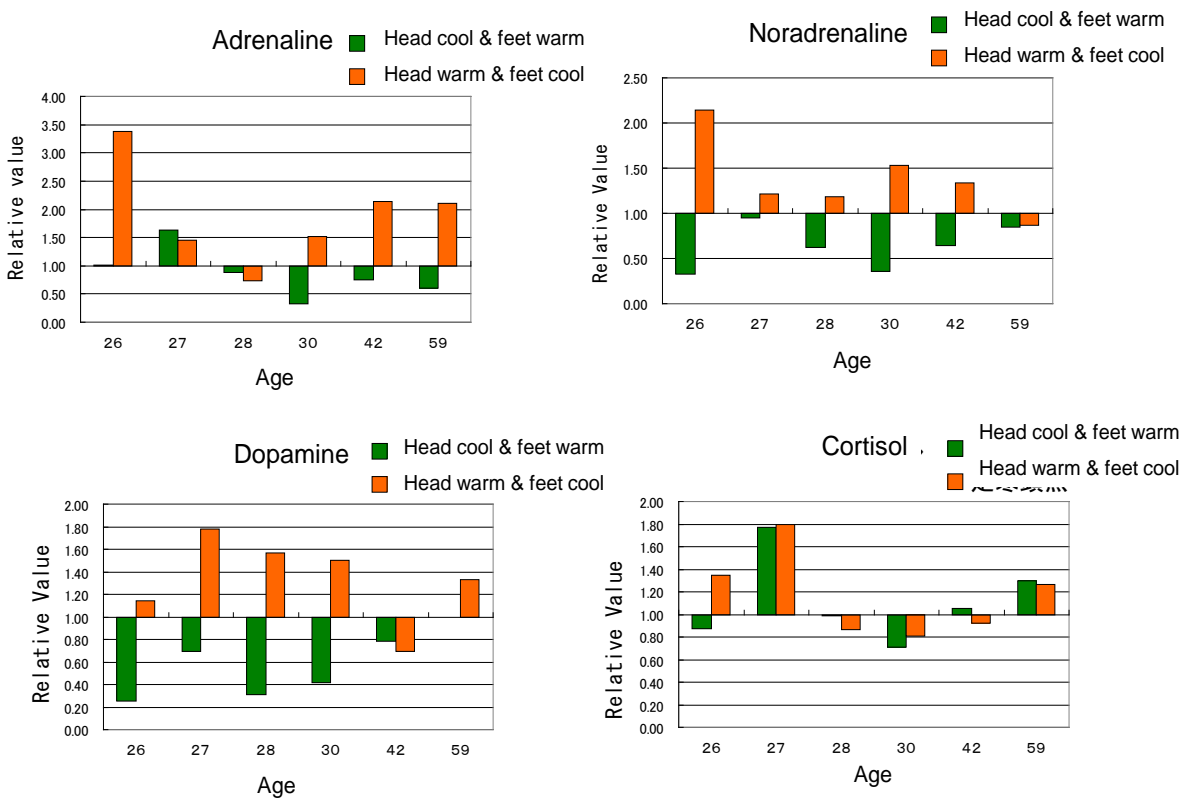

Figure 31. We tried to express the effect of peripheral total leukocyte number by individual level of change and plot in the $\mathrm{x}$-axis as in each value according to the age. The relative value (\%) of post trial was calculated with before and after and plotted in the Y-axis. Leukocyte subset, granulocyte and lymphocyte were also traced in the Figures. 
monocyte exhibited significant change by both.

\subsection{Leukocyte Subsets Regulation by Floor Heating}

After one-weeks of working in the conditioned room as HCFH or HHFC, we set up the different mode of trial at the same course and by the same volunteers. None of volunteer is dropped out with any serious problems. In this time, we set up HCFH system, asking to work about for 5 days. We select four hormones and access the effect of HCFH or HHFC conditioned room. After working about regular desk working in the HCFH conditioned room, each volunteer is down-regulated the adrenaline, cortisone and nor-adrenalin. However, dopamine level is up-regulated for all the individuals being tested. On the other hand, HHFC system shows a reversed effect for all the hormones in this text [21] [57] (Figure 31).

\section{References}

[1] Kurashige, S., Yoshida, T. and Mitsuhashi, S. (1980) Immune Response in Sarcoma 10-Bearing Mice. Annual Report of Gunnma University, 1, 36-44.

[2] Miyazaki, S. (1977) Immunodificiency in Clinical Origin. Clinical Pediatrics, 1, 1001-1006.

[3] Kishida, K., Miyazaku, S., Take, H., Fujimoto, T., Shi, H., Sasaki, K. and Goya, N. (1978) Granial Irradiation and Lymphocyte Subpopulation in Acute Lymphatic Leukemia. Journal of Pediatrics, 92, 785-786. http://dx.doi.org/10.1016/S0022-3476(78)80155-3

[4] Yamaguchi, N., Takei, T., Chen, R., Wushuer, P. and Wu, H.W. (2013) Maternal Bias of Immunity to Her Offspring: Possibility of an Autoimmunity Twist out from Maternal Immunity to Her Young. Open Journal of Rheumatology and Autoimmune Diseases, 3, Article ID: 28147.

[5] Murgita, R.A. and Tomasi Jr., T.B. (1975) Suppression of the Immune Response by Alpha-Fetoprotein. The Journal of Experimental Medicine, 141, 269-286. http://dx.doi.org/10.1084/jem.141.2.269

[6] Paul, G., Margaret, S., Liew, Y.F. and Allan, M.M. (1995) CD4 ${ }^{+}$but Not CD8 ${ }^{+}$T Cells Are Required for the Induction of Oral Tolerance. International Immunology, 7, 501-504. http://dx.doi.org/10.1093/intimm/7.3.501

[7] Koshimo, H., Miyazawa, H.Y., Shimizu, Y. and Yamaguchi, N. (1989) Maternal Antigenic Stimulation Actively Produces Suppressor Activity in Offspring. Developmental \& Comparative Immunology, 13, 79-85. http://dx.doi.org/10.1016/0145-305X(89)90020-7

[8] Zoeller, M. (1988) Tolerization during Pregnancy: Impact on the Development of Antigen-Specific Help and Suppression. European Journal of Immunology, 18, 1937-1943. http://dx.doi.org/10.1002/eji.1830181211

[9] Auerback, R. and Clark, S. (1975) Immunological Tolerance: Transmission from Mother to Offspring. Science, 189, 811-813. http://dx.doi.org/10.1126/science.1162355

[10] Shinka, S., Dohi, Y., Komatsu, T., Natarajan, R. and Amano, T. (1974) Immunological Unresponsiveness in Mice. I. Immunological Unresponsiveness Induced in Embryonic Mice by Maternofetal Transfer of Human-Globulin. Biken Journal, 17, 59-72.

[11] Aase, J.M., Noren, G.R., Reddy, D.V. and Geme Jr., J.W. (1972) Mumps-Virus Infection in Pregnant Women and the Immunologic Response of Their Offspring. The New England Journal of Medicine, 286, 1379-1382. http://dx.doi.org/10.1056/NEJM197206292862603

[12] Cramer, D.V., Kunz, H.W. and Gill III, T.J. (1974) Immunologic Sensitization Prior to Birth. American Journal of Obstetrics \& Gynecology, 120, 431-439.

[13] Wang, X.X., Kitada, K., Matsui, S., Ohkawa, T., Sugiyama, H., Kohno, S., Shimizu, S., Lai, J.-., Matsuno, H. and Yamaguchi, N. (1999) Variation of Cell Populations Taking Charge of Immunity in Human Peripheral Blood Following Hot Spring Hydrotherapy Quantitative Discussion. The Journal of Japanese Association of Physical Medicine, Balneology and Climatology, 62, 129-134.

[14] Matsuno, H., Wang, X.X., Wan, W., Matsui, K., Ohkawa, S., Sugiyama, T., Kohno, H., Shimizu, S., Lai, J.-E. and Yamaguchi, N. (1999) Variation of Cell Populations Taking Charge of Immunity in Human Peripheral Blood Following Hot Spring Hydrotherapy Qualitative Discussion. The Journal of Japanese Association of Physical Medicine, Balneology and Climatology, 62, 135-140.

[15] Yamaguchi, N., Hashimoto, H., Arai, M., Takada, S., Kawada, N., Taru, A., Li, A.L., Izumi, H. and Sugiyama, K. (2002) Effect of Acupuncture on Leukocyte and Lymphocyte Subpopulation in Human Peripheral Blood-Quantitative Discussion. The Journal of Japanese Association of Physical Medicine, Balneology and Climatology, 65, 199-206.

[16] Wan, W., Li, A.L., Izumi, H., Kawada, N., Arai, M., Takada, A., Taru, A., Hashimoto, H. and Yamaguchi, N. (2002) Effect of Acupuncture on Leukocyte and Lymphocyte Sub-Population in Human Peripheral Blood Qualitative Discus- 
sion. The Journal of Japanese Association of Physical Medicine, Balneology and Climatology, 65, 207-211.

[17] Wang, X.X., Katoh, S. and Liu, B.X. (1998) Effect of Physical Exercise on Leukocyte and Lymphocyte Subpopulations in Human Peripheral Blood. Cytometry Research, 8, 53-61.

[18] Kitada, Y., Wan, W., Matsui, K., Shimizu, S. and Yamaguchi, N. (2000) Regulation of Peripheral White Blood Cells in Numbers and Functions through Hot-Spring Bathing during a Short Term Studies in Control Experiments. Journal of Japanese Society Balneology Climatology Physiological Medicine, 63, 151-164.

[19] Yamaguchi, N., Takahashi, T., Sugita, T., Ichikawa, K., Sakaihara, S., Kanda, T., Arai, M. and Kawakita, K. (2007) Acupuncture Regulates Leukocyte Subpopulations in Human Peripheral Blood. Evidence-Based Complementary and Alternative Medicine, 4, 447-453. http://dx.doi.org/10.1093/ecam/nel107

[20] Yamaguchi, N., Shimizu, S. and Izumi, H. (2004) Hydrotherapy Can Modulate Peripheral Leukocytes: An Approach to Alternative Medicine, Complementary and Alternative Approaches to Biomedicine. Ishikawa, 239-251.

[21] Yamaguchi, N., Chen, R., Okamoto, K., Takei, T., Tsubokawa, M., Sakamoto, D., Jia, X.F., Wan, W. and Murayama, T. (2013) Quantitative Regulation of Peripheral Leukocyte by Light Exercise and Tailored Scale for Assessment. Open Journal of Immunology, 3, 175-183.

[22] Yamaguchi, N., Wan, W., Sakamoto, D., Nurmuhammad, A., Matsumoto, K., Takei, T., Okuzumi, K., Murayama, T. and Takahashi T. (2013) Regulative Effect for Natural Killer Cell by Hot Spring Hydrotherapy—Quantitative and Qualitative Discussion. Open Journal of Immunology, 3, 201-209.

[23] Abo, T., Kawate, T., Itoh, K. and Kumagai, K. (1981) Studies on the Bioperiodicity of the Immune Response. 1. Circadian Rhythms of Human T, B and K Cell Traffic in the Peripheral Blood. Journal of Immunology, 126, 1360-1363.

[24] Abo, T. and Kumagai, T. (1978) Studies of Surface Immunoglobulins on Human B Lymphocytes. III Physiological Variations of Sig+ Cells in Peripheral Blood. Clinical Experimental Immunology, 33, 441-452.

[25] Suzuki, S., Toyabe, S., Moroda, T., Tada, T., Tsukahara, A. and Iiai, T. (1997) Circadian Rhythm of Leukocytes and Lymphocytes Subsets and Its Possible Correlation with the Function of the Autonomic Nervous System. Clinical Experimental Immunology, 110, 500-508. http://dx.doi.org/10.1046/j.1365-2249.1997.4411460.x

[26] Bylund, D.B., Eikenberg, D.C., Hieble, J.P., Langer, S.Z., Lefkowitz, R.J. and Minneman, K.P. (1994) Intenational Union of Pharmacology Nomenclature of Adrenoceptors. Pharmacological Review, 46, 121-136.

[27] Dulis, B.H. and Wilson, I.B. (1980) The $\beta$-Adrenergic Receptor of Live Human Polymorphonuclear Leukocytes. Journal of Biological Chemistry, 255, 1043-1048.

[28] Maisel, A.S., Harris, T., Rearden, C.A. and Michel, M.C. (1990) Beta-Adrenergic Receptors in Lymphocyte Subsets after Exercise. Alterations in Normal Individuals and Patients with Congestive Heart Failure. Circulation, 82, 20032010. http://dx.doi.org/10.1161/01.CIR.82.6.2003

[29] Sanders, V.M., Baker, R.A., Ramer-Quinn, D.S., Kasprowicz, D.J., Fuchs, B.A. and Street, N.E. (1997) Differential Expression of the $\beta_{2}$-Adrenaergic Receptor by Th1 and Th2 Clones. Journal of. Immunology, 158, 4200-4210.

[30] Yamaguchi, N., Ueyama, T., Amat, N., Yimit, D., Hoxur, P., Sakamoto, D., Katoh, Y., Watanabe, I. and Su, S.Y. (2015) Bi-Directional Regulation by Chinese Herbal Formulae to Host and Parasite for Multi-Drug Resistant Staphylococcus aureus in Humans and Rodents. Open Journal of Immunology, 4, 18-32. http://dx.doi.org/10.4236/oji.2015.51003

[31] Gorantla, S., Dou, H., Boska, M., Destache, C.J., Nelson, J., Poluektova, L., Rabinow, B.E., Gendelman, H.E. and Mosley, R.L. (2006) Quantitative Magnetic Resonance and SPECT Imaging for Macrophage Tissue Migration and Nanofor-Mulated Drug Delivery. Journal of Leukocyte Biology, 80, 1165-1174. http://dx.doi.org/10.1189/jlb.0206110

[32] Stoika, R.S., Lutsik, M.D., Barska, M.L., Tsyrulnyk, A.A. and Kashchak, N.I. (2002) In Vitro Studies of Activation of Phagocytic Cells by Bioactive Peptides. Journal of Physiology and Pharmacology, 53, 675-688.

[33] Elbim, C., Pillet, S., Prevost, M.H., Preira, A., Girard, P.M., Rogine, N., Hakim, J., Israel, N. and M. Gougerot-Pocidalo, A. (2001) The Role of Phagocytes in HIV-Related Oxidative Stress. Journal of Clinical Virology, 20, 99-109.

[34] Speer, C.P., Gahr, M. and Pabst, M.J. (1986) Phagocytosis Associated Oxidative Metabolism in Human Milk Macrophages. Acta Paediatrica Scandinavica, 75, 444-451. http://dx.doi.org/10.1111/j.1651-2227.1986.tb10228.x

[35] Iqbal, M., Sharma, S.D., Okazaki, Y., Fujisawa, M. and Okada, S. (2003) Dietary Supplementation of Curcumin Enhances Antioxidant and Phase II Metabolizing Enzymes in ddY Male Mice; Possible Role in Protection against Chemical Rall Estimation of Anti-Oxidant Activity by Mammal Macrophage Carcinogenesis and Toxicity. Pharmacology \& Toxicology, 92, 33-38. http://dx.doi.org/10.1034/j.1600-0773.2003.920106.X

[36] Kohno, H., Tanaka, T., Kawabata, K., Hirose, Y., Sugi, S., Tsuba, H. and Mori, H. (2002) Silymarin, a Naturally Occurring Polyphenolic Anotioxidant Flavionoid, Inhibits Azoxymethane-Inbuced Colon Carcinogenesis in Male F344 Rats. Oxidative Stress in Dermatology, In: Fuchs, J. and Packer, L., Eds., Marcel Dekker, New York, 461-468. http://dx.doi.org/10.1002/ijc.10625 
[37] Karbownik, M., Tan, D., Manchester, L.C. and Reiter, R.J. (2000) Renal Toxicity of the Carcinogen Delta-Aminolevulinic Acid: Antioxidant Effects of Melatonin. Cancer Letters, 161, 1-7. http://dx.doi.org/10.1016/S0304-3835(00)00568-1

[38] Harman, D. (1956) Aging: A Theory Based on Free Radical and Radiation Chemistry. Journal of Gerontology, 11, 293-300. http://dx.doi.org/10.1093/geronj/11.3.298

[39] Inoue, M. (2002) Free Radical Theory of Aging. Nippon Ronen Igakkai Zasshi, 39, 36-38.

[40] Biesalski, H.K. (2002) Free Radical Theory of Aging. Current Opinion in Clinical Nutrition and Metabolic Care, 5, 510. http://dx.doi.org/10.1097/00075197-200201000-00002

[41] Wei, Y.H. and Lee, H.C. (2002) Oxidative Stress, Mitochondrial DNA Mutation, and Impairment of Antioxidant Enzymes in Aging. Experimental Biology and Medicine (Maywood), 227, 671-682.

[42] Sanz, N., Diez-Fernandez, C., Andres, D. and Cascales, M. (2002) Hepatotoxicity and Aging: Endogenous Antioxidant Systems in Hepatocytes from 2-, 6-, 12-, 18- and 30- Month-Old Rats Following a Necrogenic Dose of Thioacetamide. Biochimica et Biophysica Acta, 1587, 12-20. http://dx.doi.org/10.1016/S0925-4439(02)00048-0

[43] Diamond, J., Skaggs, J. and Manaligod, J.M. (2002) Free-Radical Damage: A Possible Mechanism of Laryngeal Aging. Ear, Nose \& Throat, 81, 531-533.

[44] Melov, S. (2002) Animal Models of Oxidative Stress, Aging, and Therapeutic Antioxidant Interventions. International Journal of Biochemistry \& Cell Biology, 34, 1395-1400. http://dx.doi.org/10.1016/S1357-2725(02)00086-9

[45] Arockia-Rani, P.J. and Panneerselvam, C. (2001) Carnitine as a Free Radical Scavenger in Aging. Experimental Gerontology, 36, 1713-1726.

[46] Kasapoglu, M. and Ozben, T. (2001) Alterations of Antioxidant Enzymes and Oxidative Stress Markers in Aging. Experimental Gerontology, 36, 209-220. http://dx.doi.org/10.1016/S0531-5565(00)00198-4

[47] Perchellet, J.P. and Perchellet, M. (1989) Antioxidants and Multistage Carcingenesis in Mouse Skin. Free Radical Biology and Medicine (Medsci), 7, 377-408. http://dx.doi.org/10.1016/0891-5849(89)90124-X

[48] Edsmyr, F. (1982) Super Oxide Anion Dismutase Efficacy in Ameliorating Side Effects of Radiation Therapy. Pathology of Oxygen, Academic Press, New York, 315-326.

[49] Johansson, M.H., Deinum, J., Marklund, S.L. and Sjoquist, P.O. (1990) Recombinant Human Extra-Celluar Super Oxide Anion Dismutase Reduces Concentration of Oxygen Free Redicals in the Reperfusedret Heart. Cardiouas, 24, 500-503.

[50] Venugopal, S.K., Devaraj, S., Yang, T. and Jialal, L. (2002) Alpha-Tocopherol Decreases Superoxide Anion Release in Human Monocytes under Hyperglycemic Conditions via Inhibition of Protein Kinase C-Alpha. Diabetes, 51, 30493054. http://dx.doi.org/10.2337/diabetes.51.10.3049

[51] Nayak, D.U., Karmen, C., Frishman, W.H. and Va-kili, B.A. (2001) Antioxidant Vitamins and Enzymatic and Synthetic Oxygen-Derived Free Radical Scavengers in the Prevention and Treatment of Cardiovascular Disease. Heart Disease, 3, 28-45. http://dx.doi.org/10.1097/00132580-200101000-00006

[52] Miyachi, Y. (1993) Skin Diseases Associated with Oxidative Injury. Oxidative Stress in Dermatology, 323-331.

[53] De Beer, D., Joubert, E., Gelderblom, W.C. and Manley, M. (2003) Antioxidant Activity of South African Red and White Cultivar Wines: Free Radical Scavenging. Journal of Agricultural and Food Chemistry, 51, 902-909. http://dx.doi.org/10.1021/jf0260110

[54] Lee, S.E., Ju, E.M. and Kim, J.H. (2001) Free Radical Scavenging and Antioxidant Enzyme Fortifying Activities of Extracts from Smilax China Root. Experimental and Molecular Medicine, 33, 263-268. http://dx.doi.org/10.1038/emm.2001.43

[55] Joshi, R., Adhikari, S., Patro, B.S., Chattopadhyay, S. and Mukherjee, T. (2001) Free Radical Scavenging Behavior of Folic Acid: Evidence for Possible Antioxidant Activity. Free Radical Biology \& Medicine, 30, 1390-1399. http://dx.doi.org/10.1016/S0891-5849(01)00543-3

[56] Lin, C.C., Hsu, Y.F. and Lin, T.C. (2001) Antioxidant and Free Radical Scavenging Effects of the Tannins of Terminalia catappa L. Anticancer Research, 21, 237-243.

[57] Shimizu, S., Kitada, H., Yokota, H., Yamakawa, J., Murayama, T., Sugiyama, K., Izumi, H. and Yamaguchi, N. (2002) Activation of the Alternative Complement Pathway by Agaricus Blazei Murill. Phytomedicine, 9, 536-545. 